\title{
T-Cell Cytokine Gene Polymorphisms and Vitamin D Pathway Gene Polymorphisms in End-Stage Renal Disease due to Type 2 Diabetes Mellitus Nephropathy: Comparisons with Health Status and Other Main Causes of End-Stage Renal Disease
}

\author{
Alicja E. Grzegorzewska, ${ }^{1}$ Grzegorz Ostromecki, ${ }^{2}$ Paulina Zielińska, ${ }^{3}$ \\ Adrianna Mostowska, ${ }^{4}$ and Paweł P. Jagodziński ${ }^{4}$ \\ ${ }^{1}$ Department of Nephrology, Transplantology and Internal Diseases, Poznań University of Medical Sciences (PUMS), \\ 49 Przybyszewskiego Boulevard, 60-355 Poznań, Poland \\ ${ }^{2}$ DaVita Clinic Piła Dialysis Center, Wojska Polskiego 43, 64-420 Piła, Poland \\ ${ }^{3}$ Student Nephrology Research Group, Department of Nephrology, Transplantology and Internal Diseases, PUMS, \\ Przybyszewskiego 49, 60-355 Poznań, Poland \\ ${ }^{4}$ Department of Biochemistry and Molecular Biology, PUMS, Święcickiego 6, 60-781 Poznań, Poland
}

Correspondence should be addressed to Alicja E. Grzegorzewska; alicja_grzegorzewska@yahoo.com

Received 17 July 2014; Revised 22 September 2014; Accepted 22 September 2014; Published 22 December 2014

Academic Editor: Salwa Ibrahim

Copyright (C) 2014 Alicja E. Grzegorzewska et al. This is an open access article distributed under the Creative Commons Attribution License, which permits unrestricted use, distribution, and reproduction in any medium, provided the original work is properly cited.

\begin{abstract}
Background. T-cell cytokine gene polymorphisms and vitamin D pathway gene polymorphisms were evaluated as possibly associated with end-stage renal disease (ESRD) resulting from type 2 diabetes mellitus (DM) nephropathy. Methods. Studies were conducted among hemodialysis (HD) patients with ESRD due to type 2 DM nephropathy, chronic glomerulonephritis, chronic infective tubulointerstitial nephritis, and hypertensive nephropathy as well as in healthy subjects. A frequency distribution of T-cellrelated interleukin (IL) genes (IL18 rs360719, IL12A rs568408, IL12B rs3212227, IL4R rs1805015, IL13 rs20541, IL28B rs8099917, IL28B, and rs12979860) and vitamin D pathway genes (GC genes: rs2298849, rs7041, and rs1155563; VDR genes: rs2228570, rs1544410; and RXRA genes: rs10776909, rs10881578, and rs749759) was compared between groups. Results. No significant differences in a frequency distribution of tested polymorphisms were shown between type $2 \mathrm{DM}$ nephropathy patients and controls. A difference was found in IL18 rs360719 polymorphic distribution between the former group and chronic infective tubulointerstitial nephritic patients $\left(P_{\text {trend }}=0.033\right)$, which also differed in this polymorphism from controls $\left(P_{\text {trend }}=0.005\right)$. Conclusion. T-cell cytokine and vitamin D pathway gene polymorphisms are not associated with ESRD due to type 2 DM nephropathy in Polish HD patients. IL18 rs360719 is probably associated with the pathogenesis of chronic infective tubulointerstitial nephritis.
\end{abstract}

\section{Introduction}

Diabetes mellitus (DM) is the most common cause of endstage renal disease (ESRD) in many hemodialysis (HD) centers. In Australia and New Zealand, the incident ESRD population (1991-2005) who began renal replacement therapy (RRT) included $30.0 \%$ type $2 \mathrm{DM}$ and $4.5 \%$ type $1 \mathrm{DM}$ subjects [1]. In the HEMODIALYSIS (HEMO) study, the group of HD patients comprised approximately $45 \%$ of DM subjects [2].
Diabetic ESRD patients compared to nondiabetic ESRD subjects show higher both mortality rate [3] and prevalence of coronary artery disease (CAD) [4], are more prone to severe infections [5] and worse response to hepatitis $B$ vaccination [6], and more often suffer from adynamic bone disease associated with low serum parathyroid hormone (PTH) levels [7]. In this paper we will focus on ESRD due to type 2 DM nephropathy. Together with altered glucose metabolism and insulin resistance, deficiency of vitamin $\mathrm{D}$ [8] and aberrant T-cell cytokine balance [9] were found to be associated with 


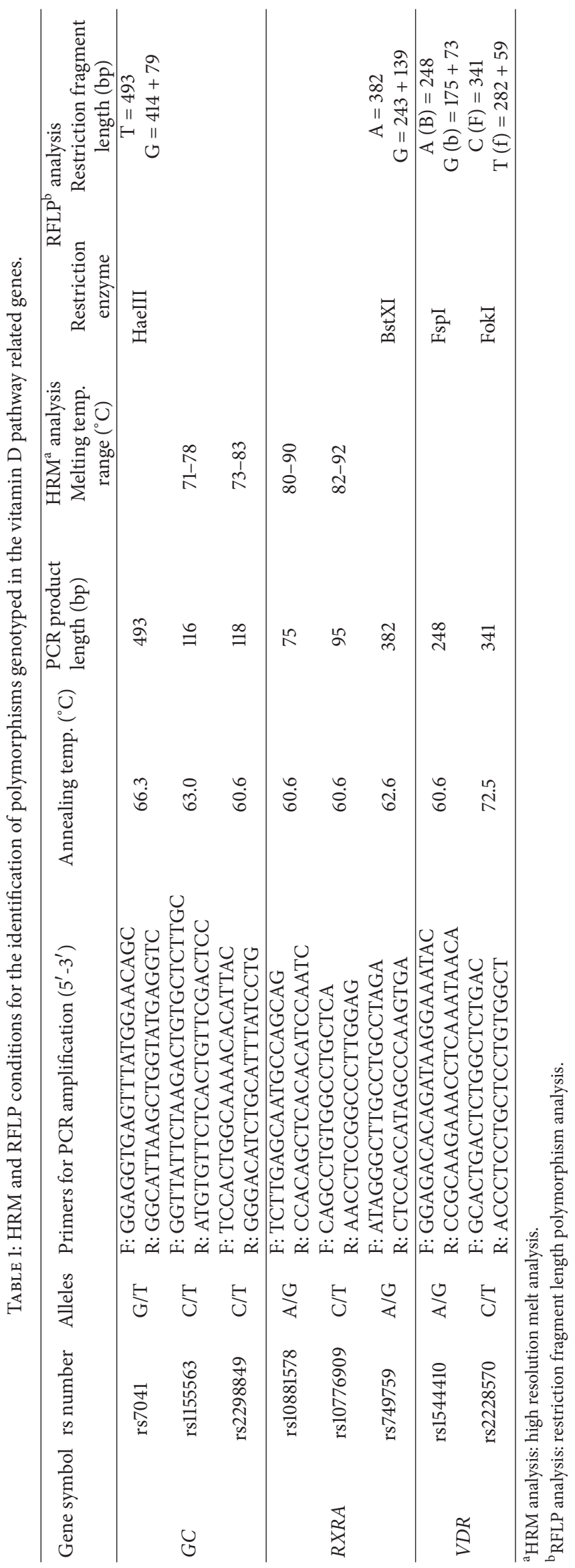


TABLE 2: Characteristics of hemodialysis patients $(n=893)$.

\begin{tabular}{|c|c|c|c|}
\hline Parameter & Type 2 DM nephropathy & Other causes of ESRD & $P$ value \\
\hline Demographic data & $n=366$ & $n=527$ & \\
\hline Male sex, $n(\%$ of all $)$ & $201(54.9)$ & $307(58.3)$ & $0.337^{\mathrm{b}}$ \\
\hline Age at RRT beginning, years & $62.9 \pm 14.1$ & $57.2 \pm 17.2$ & $<0.0001^{c}$ \\
\hline RRT duration, years & $3.29(0.06-28.0)$ & $4.42(0.12-28.2)$ & $<0.0001^{\mathrm{c}}$ \\
\hline Death rate, cases per 100 patient-years & 0.48 & 0.42 & \\
\hline Death rate, cases per 100 RRT-years & 7.97 & 4.63 & \\
\hline Clinical data & $n=332$ & $n=527$ & \\
\hline Coronary artery disease, $n$ ( $\%$ of all) & $174(52.4)$ & $168(31.9)$ & $<0.0001^{\mathrm{b}}$ \\
\hline Myocardial infarction, $n$ (\% of all) & $98(29.5)$ & $101(19.2)$ & $0.009^{\mathrm{b}}$ \\
\hline Parathyroidectomy, $n$ (\% of all) & $2(0.60)$ & $21(3.98)$ & $0.0009^{b}$ \\
\hline Treatment with cinacalcet hydrochloride & $24(7.2)$ & $98(18.6)$ & $<0.0001^{\text {b }}$ \\
\hline Laboratory data & $n=366$ & $n=527$ & \\
\hline Anti-HBc positive, $n$ (\% of all) & $95(26.0)$ & $126(23.9)$ & $0.528^{\mathrm{b}}$ \\
\hline HBsAg positive, $n$ (\% of all anti-HBc positive) & $7(7.4)$ & $11(8.7)$ & $0.807^{\mathrm{b}}$ \\
\hline Anti-HCV positive, $n$ ( $\%$ of all) & $26(7.1)$ & $57(10.8)$ & $0.062^{\mathrm{b}}$ \\
\hline HCV RNA positive, $n$ ( $\%$ of all anti-HCV positive) & $14(53.8)$ & $39(68.4)$ & $0.225^{\mathrm{b}}$ \\
\hline Responders to hepatitis B vaccine, $n$ (\% of all) & $202(55.2)$ & $315(59.8)$ & $0.191^{\mathrm{b}}$ \\
\hline $25(\mathrm{OH}) \mathrm{D}(\mathrm{ng} / \mathrm{mL})^{\mathrm{a}}$ & $13.3 \pm 3.9$ & $14.5 \pm 5.6$ & $0.182^{\mathrm{a}, \mathrm{d}}$ \\
\hline Total calcium (mg/dL) & $8.83 \pm 0.67$ & $8.91 \pm 0.82$ & $0.264^{\mathrm{d}}$ \\
\hline Phosphates (mg/dL) & $5.03 \pm 1.44$ & $5.25 \pm 1.49$ & $0.054^{\mathrm{d}}$ \\
\hline PTH $(\mathrm{pg} / \mathrm{mL})$ & $296(12.9-3,757)$ & $463(12.7-3,741)$ & $<0.0001^{\mathrm{c}}$ \\
\hline Total alkaline phosphatase (U/L) & $98.2(25.8-1,353)$ & $97.1(40.5-1,684)$ & $0.528^{\mathrm{c}}$ \\
\hline \multicolumn{4}{|c|}{$\begin{array}{l}\text { 25(OH)D: } 25 \text {-hydroxycholecalciferol, anti-HBc: antibodies to core antigen of hepatitis B virus, anti-HCV: antibodies to hepatitis C virus, HBsAg: surface antigen } \\
\text { of hepatitis B virus, DM: diabetes mellitus, ESRD: end-stage renal disease, HCV RNA: ribonucleic acid of hepatitis C virus, PTH: parathyroid hormone, and } \\
\text { RRT: renal replacement therapy. } \\
\text { A significant difference is indicated using bold font. } \\
\text { a } n=66 \text { for type } 2 \text { DM nephropathy; } n=96 \text { for other renal diseases. } \\
\text { bisher's exact test. }\end{array}$} \\
\hline
\end{tabular}

this severe complication of type $2 \mathrm{DM}$. There is a link between vitamin $\mathrm{D}$ and $\mathrm{T}$-cell functional balance: active form of vitamin $\mathrm{D}\left[1,25(\mathrm{OH})_{2} \mathrm{D}\right]$ has the inhibitory effect on the $\mathrm{T}$ helper (Th) 17 and Th1 response [10].

Abnormalities in T-cell cytokine equilibrium [11-13] and plasma vitamin D concentrations [14-16] are related to cardiovascular events $[13,16]$ and immunononcompetence during infections [11, 14] and vaccinations [12, 15]. Serum PTH levels are dependent on serum vitamin D concentrations [17], and T cells are implicated in the mechanism of PTH action in bone [18].

Vitamin D activity may be adequately expressed if vitamin D pathway components (vitamin D binding protein, also referred to as group-specific component (GC), vitamin $\mathrm{D}$ receptor (VDR), and retinoid $\mathrm{X}$ receptors (RXRs)) are properly structured and regulated. The recent study by Zhang et al. [19] has shown that VDR BsmI polymorphism correlates with type 2 DM nephropathy and may be susceptible for early onset of this nephropathy. Among T-cell-related cytokine gene polymorphisms, promoter polymorphic variants of IL10 [20, 21] and IL6 [22] were already associated with the risk of type 2 DM nephropathy. Monocyte chemoattractant protein 1 (MCP-1) has been reported to participate in the pathogenesis of early type $2 \mathrm{DM}$ nephropathy [23], but $M C P 1$ polymorphism in the promoter region was not differentially distributed between ESRD patients with type $2 \mathrm{DM}$ nephropathy and healthy controls $[24,25]$.

To our knowledge, there are scarce data, if any, on ESRD due to type 2 DM nephropathy showing a frequency distribution of single nucleotide polymorphisms (SNPs) of T-cell-related IL genes: IL18 rs360719, IL12A rs568408, IL12B rs3212227, IL4R rs1805015, IL13 rs20541, IL28B rs8099917, and $I L 28 B$ rs12979860 as well as vitamin D pathway genes: GC genes (GC rs2298849, rs7041, and rs1155563), VDR genes ( VDR rs2228570, rs1544410), and RXR $\alpha$ genes ( $R X R A$ rs10776909, rs10881578, and rs749759). The aim of our study was to determine the potential association between aforementioned polymorphisms of T-cell-related cytokine genes and vitamin $\mathrm{D}$ pathway genes and ESRD due to type $2 \mathrm{DM}$ nephropathy. For comparisons, aforementioned genotype frequencies of healthy controls as well as ESRD patients with other main causes of ESRD were used. Polymorphism related associations, if exist, could contribute to explanation of susceptibility to ESRD due to type 2 DM nephropathy and phenotype differences between ESRD patients with type 2 DM nephropathy and other causes of ESRD. 
TABLE 3: Characteristics of hemodialysis patients grouped by a cause of ESRD.

\begin{tabular}{|c|c|c|c|c|c|}
\hline Parameter & $\begin{array}{c}\text { Type } 2 \text { DM } \\
\text { nephropathy (1) }\end{array}$ & $\begin{array}{c}\text { Chronic } \\
\text { glomerulonephritis } \\
(2)\end{array}$ & $\begin{array}{c}\text { Chronic } \\
\text { tubulointerstitial } \\
\text { nephritis (3) }\end{array}$ & $\begin{array}{c}\text { Hypertensive } \\
\text { nephropathy (4) }\end{array}$ & $P$ value \\
\hline Demographic data & $n=366$ & $n=178$ & $n=118$ & $n=231$ & \\
\hline Male sex, $n$ (\% of all) & $201(54.9)$ & $110(61.8)$ & $63(53.4)$ & $134(58.0)$ & $0.386^{\mathrm{b}}$ \\
\hline $\begin{array}{l}\text { Age at RRT beginning, } \\
\text { years }\end{array}$ & $62.9 \pm 14.1$ & $47.4 \pm 17.6$ & $59.9 \pm 16.6$ & $63.3 \pm 13.6$ & $\begin{array}{l}\quad<0.0001^{\mathrm{c}} \\
1 \text { versus } 2:<0.001^{\mathrm{c}} \\
2 \text { versus } 3:<0.001^{\mathrm{c}} \\
2 \text { versus } 4:<0.001^{\mathrm{c}}\end{array}$ \\
\hline RRT duration, years & $3.29(0.06-28.0)$ & $5.73(0.16-28.2)$ & $4.82(0.33-26.5)$ & $3.82(0.12-20.4)$ & $\begin{array}{c}<0.0001^{\mathrm{c}} \\
1 \text { versus } 2:<0.001^{\mathrm{c}} \\
1 \text { versus } 3:<0.01^{\mathrm{c}} \\
2 \text { versus } 4:<0.001^{\mathrm{c}}\end{array}$ \\
\hline $\begin{array}{l}\text { Death rate, cases per } 100 \\
\text { patient-years }\end{array}$ & 0.48 & 0.41 & 0.44 & 0.42 & \\
\hline $\begin{array}{l}\text { Death rate, cases per } 100 \\
\text { dialysis-years }\end{array}$ & 7.97 & 2.87 & 5.28 & 6.70 & \\
\hline Clinical data & $n=332$ & $n=178$ & $n=118$ & $n=231$ & \\
\hline $\begin{array}{l}\text { Coronary artery disease, } \\
n \text { ( } \% \text { of all) }\end{array}$ & $174(52.4)$ & $43(24.2)$ & $29(24.6)$ & $96(41.5)$ & $\begin{array}{c}<0.0001^{\mathrm{b}} \\
1 \text { versus } 2:<0.0001^{\mathrm{e}} \\
1 \text { versus } 3:<0.0001^{\mathrm{e}} \\
1 \text { versus } 4: 0.013^{\mathrm{e}} \\
2 \text { versus } 4: 0.0002^{\mathrm{e}} \\
3 \text { versus } 4: 0.002^{\mathrm{e}}\end{array}$ \\
\hline $\begin{array}{l}\text { Myocardial infarction, } \\
n \text { (\% of all) }\end{array}$ & $98(29.5)$ & $25(14.0)$ & $17(14.4)$ & $59(25.5)$ & $\begin{array}{c}<0.0001^{\mathrm{b}} \\
1 \text { versus } 2:<0.0001^{\mathrm{e}} \\
1 \text { versus } 3:<0.0001^{\mathrm{e}} \\
1 \text { versus } 4:<0.0001^{\mathrm{e}} \\
2 \text { versus } 4: 0.005^{\mathrm{e}} \\
3 \text { versus } 4: 0.02^{\mathrm{e}}\end{array}$ \\
\hline PTX, $n$ (\% of all) & $2(0.60)$ & $14(7.9)$ & $5(4.2)$ & $2(0.87)$ & $\begin{array}{c}<0.0001^{\mathrm{b}} \\
1 \text { versus } 2:{<0.0001^{\mathrm{e}}}^{\mathrm{e}} \\
1 \text { versus } 3: 0.015^{\mathrm{e}} \\
\text { 2 versus } 4: 0.0004^{\mathrm{e}} \\
3 \text { versus } 4: 0.046^{\mathrm{e}}\end{array}$ \\
\hline $\begin{array}{l}\text { Treatment with cinacalcet } \\
\text { hydrochloride }\end{array}$ & $24(7.2)$ & $48(27.0)$ & $21(17.8)$ & $29(12.6)$ & $\begin{array}{c}<0.0001^{\mathrm{b}} \\
1 \text { versus } 2:<0.0001^{\mathrm{e}} \\
1 \text { versus 3: } 0.0008^{\mathrm{e}} \\
1 \text { versus } 4: 0.017^{\mathrm{e}} \\
2 \text { versus } 4: 0.0003^{\mathrm{e}}\end{array}$ \\
\hline Laboratory data & $n=366$ & $n=178$ & $n=118$ & $n=231$ & \\
\hline $\begin{array}{l}\text { Anti-HBc positive, } \\
n \text { ( } \% \text { of all) }\end{array}$ & $95(26.0)$ & $53(29.8)$ & $25(21.2)$ & $48(20.8)$ & $0.233^{\mathrm{b}}$ \\
\hline $\begin{array}{l}\text { HBsAg positive, } \\
n(\% \text { of all anti-HBc } \\
\text { positive })\end{array}$ & $7(7.4)$ & $10(18.9)$ & $0(0.0)$ & $1(2.08)$ & $\begin{array}{c}0.0007^{\mathrm{b}} \\
1 \text { versus } 2: 0.032^{\mathrm{e}} \\
2 \text { versus 3: } 0.007^{\mathrm{e}} \\
2 \text { versus } 4: 0.001^{\mathrm{e}}\end{array}$ \\
\hline $\begin{array}{l}\text { Anti-HCV positive, } \\
n(\% \text { of all })\end{array}$ & $26(7.1)$ & $33(18.5)$ & $11(9.3)$ & $13(5.6)$ & $\begin{array}{c}<0.0001^{\mathrm{b}} \\
1 \text { versus } 2: 0.0004^{\mathrm{e}} \\
2 \text { versus } 3: 0.031^{\mathrm{e}} \\
2 \text { versus } 4:<0.0001^{\mathrm{e}}\end{array}$ \\
\hline $\begin{array}{l}\text { HCV RNA positive, } \\
n \text { (\% of all anti-HCV } \\
\text { positive) }\end{array}$ & $14(53.8)$ & $27(81.8)$ & $4(36.4)$ & $8(61.5)$ & $\begin{array}{c}<0.0001^{\mathrm{b}} \\
1 \text { versus } 2:<0.0001^{\mathrm{e}} \\
2 \text { versus } 3: 0.0004^{\mathrm{e}} \\
2 \text { versus } 4:<0.0001^{\mathrm{e}}\end{array}$ \\
\hline
\end{tabular}


TABLE 3: Continued.

\begin{tabular}{|c|c|c|c|c|c|}
\hline Parameter & $\begin{array}{c}\text { Type } 2 \text { DM } \\
\text { nephropathy (1) }\end{array}$ & $\begin{array}{c}\text { Chronic } \\
\text { glomerulonephritis } \\
(2)\end{array}$ & $\begin{array}{c}\text { Chronic } \\
\text { tubulointerstitial } \\
\text { nephritis (3) }\end{array}$ & $\begin{array}{c}\text { Hypertensive } \\
\text { nephropathy (4) }\end{array}$ & $P$ value \\
\hline $\begin{array}{l}\text { Responders to hepatitis B } \\
\text { vaccine, } n \text { ( } \% \text { of all) }\end{array}$ & $202(55.2)$ & $107(60.1)$ & $70(59.3)$ & $138(59.7)$ & $0.598^{\mathrm{b}}$ \\
\hline $25(\mathrm{OH}) \mathrm{D}(\mathrm{ng} / \mathrm{mL})^{\mathrm{a}}$ & $13.3 \pm 3.9$ & $14.2 \pm 7.3$ & $15.7 \pm 4.3$ & $14.1 \pm 3.9$ & $0.453^{\mathrm{d}}$ \\
\hline Total calcium (mg/dL) & $8.83 \pm 0.67$ & $8.85 \pm 0.85$ & $9.04 \pm 0.61$ & $8.88 \pm 0.87$ & $0.239^{\mathrm{d}}$ \\
\hline Phosphates (mg/dL) & $5.03 \pm 1.44$ & $5.63 \pm 1.59$ & $4.92 \pm 1.29$ & $5.15 \pm 1.47$ & $\begin{array}{c}0.0007^{\mathrm{d}} \\
1 \text { versus } 2:<0.001^{\mathrm{c}} \\
2 \text { versus } 3:<0.01^{\mathrm{c}} \\
2 \text { versus } 4:<0.05^{\mathrm{c}}\end{array}$ \\
\hline PTH (pg/mL) & $296(12.9-3,757)$ & $632(12.7-3,118)$ & $426(45.8-3,741)$ & $364(19.5-2,351)$ & $\begin{array}{c}<0.0001^{\mathrm{c}} \\
1 \text { versus } 2:<0.001^{\mathrm{c}} \\
1 \text { versus } 3:<0.05^{\mathrm{c}} \\
1 \text { versus } 4:<0.05^{\mathrm{c}} \\
2 \text { versus } 4:<0.001^{\mathrm{c}}\end{array}$ \\
\hline Total ALP (U/L) & $98.2(25.8-1,353)$ & $113(44.5-860)$ & $89.0(40.5-1,684)$ & $90.9(41.0-1,110)$ & $\begin{array}{c}0.010^{\mathrm{c}} \\
2 \text { versus } 4:<0.05^{\mathrm{c}}\end{array}$ \\
\hline
\end{tabular}

25(OH)D: 25-hydroxycholecalciferol, anti-HBc: antibodies to core antigen of hepatitis B virus, anti-HCV: antibodies to hepatitis C virus, $\mathrm{HBs} A g$ : surface antigen of hepatitis B virus, DM: diabetes mellitus, ESRD: end-stage renal disease, HCV RNA: ribonucleic acid of hepatitis C virus, PTH: parathyroid hormone, and RRT: renal replacement therapy.

a $n=66$ for type 2 DM nephropathy, $n=40$ for chronic glomerulonephritis, $n=13$ for chronic interstitial nephritis, and $n=43$ for hypertensive nephropathy.

${ }^{\mathrm{b}}$ Chi squared test.

${ }^{\mathrm{c}}$ Kruskal-Wallis test.

dANOVA test.

${ }^{\mathrm{e}}$ Fisher's exact test.

\section{Material and Methods}

2.1. Patients and Controls. Blood samples for genotype analyses are collected since 2009 from ESRD patients (estimated glomerular filtration rate (eGFR) category G5 in accordance with KDIGO recommendations [26]). All subjects were treated with HD on enrolment. Controls were recruited from blood donors and healthy volunteers unrelated to patients. All enrolled individuals live/lived in the Greater Poland region of Poland.

Genotyping of IL18 rs360719, IL12A rs568408, IL12B rs3212227, IL4R rs1805015, and IL13 rs20541 polymorphisms was performed in 2009-2012 using currently available material. Results had been analyzed in our previous studies in the context of responsiveness to the surface antigen of hepatitis B virus (HBsAg) using data of all (not segregated) patients [2730]. For this study, we used results of controls and patients with type 2 DM nephropathy, chronic glomerulonephritis, chronic infective tubulointerstitial nephritis, and hypertensive nephropathy.

IL28B rs8099917, IL28B rs12979860, GC rs2298849, GC rs7041, GC rs1155563, VDR rs2228570, VDR rs1544410, RXRA rs10776909, RXRA rs10881578, and RXRA rs749759 polymorphisms were analyzed in winter 2013/2014 among HD patients with ESRD $(n=893)$ due to type 2 DM nephropathy $(n=366)$, chronic glomerulonephritis $(n=178)$, chronic infective tubulointerstitial nephritis $(n=118)$, and hypertensive nephropathy $(n=231)$ as well as healthy controls $(n=378)$.
DM was not diagnosed in patients having renal diseases other than type 2 DM nephropathy.

Healthy individuals and HD patients with other renal diseases as cause of ESRD served as reference groups for a frequency distribution of tested polymorphic variants. All examined subjects were of Caucasian race.

Basic clinical and laboratory data were collected on enrolment and they are updated every year.

2.2. Genotyping. Genomic DNA for genotype analysis was isolated from peripheral blood lymphocytes by salt-out extraction procedure.

Genotyping of IL18 rs360719, IL12A rs568408, IL12B rs3212227, IL4R rs1805015, and IL13 rs20541 polymorphisms was performed as previously described [27-30].

IL28B rs8099917 and IL28B rs12979860 polymorphisms were genotyped using high-resolution melting curve analysis (HRM) on the LightCycler 480 system (Roche Diagnostics, Mannheim, Germany) with the use of 5x HOT FIREPol EvaGreen HRM Mix (Solis BioDyne, Tartu, Estonia). The PCR program consisted of an initial step at $95^{\circ} \mathrm{C}$ for $15 \mathrm{~min}$ to activate HOT FIREPol DNA polymerase, followed by 50 amplification cycles of denaturation at $95^{\circ} \mathrm{C}$ for $10 \mathrm{~s}$, annealing at $61^{\circ} \mathrm{C}$ for $10 \mathrm{~s}$, and elongation at $72^{\circ} \mathrm{C}$ for $15 \mathrm{~s}$. Amplified DNA fragments were then subjected to HRM with $0.1^{\circ} \mathrm{C}$ increments in temperatures ranging from 76 to $96^{\circ} \mathrm{C}$. Primers used for PCR with subsequent HRM analysis were as follows: rs8099917F 5'TTTGTCACTGTTCCTCCTTTTG3', rs8099917R 5'AAGACATAAAAAGCCAGCTACCA3', 
TABLE 4: Comparison of the distribution of polymorphic variants of tested genes between ESRD patients treated with hemodialysis due to type $2 \mathrm{DM}$ nephropathy and healthy subjects.

\begin{tabular}{|c|c|c|c|c|c|}
\hline Parameter & $\begin{array}{c}\text { Type } 2 \text { DM } \\
\text { nephropathy } \\
\text { (frequency) }\end{array}$ & $\begin{array}{l}\text { Healthy subjects } \\
\text { (frequency) }\end{array}$ & Odds ratio $(95 \% \mathrm{CI})$ & Two-tailed $P$ & $P_{\text {trend }}$ \\
\hline IL18 rs360719 & $n=248$ & $n=240$ & & & \\
\hline $\mathrm{TT}$ & $133(0.54)$ & $121(0.50)$ & Referent & & 0.233 \\
\hline $\mathrm{CT}$ & $102(0.41)$ & $98(0.41)$ & $0.947(0.654-1.372)$ & 0.777 & \\
\hline $\mathrm{CC}$ & $13(0.05)$ & $21(0.09)$ & $0.563(0.270-1.174)$ & 0.145 & \\
\hline $\mathrm{CT}+\mathrm{CC}$ & $115(0.46)$ & $119(0.50)$ & $0.879(0.616-1.254)$ & 0.526 & \\
\hline MAF & $128(0.26)$ & $140(0.29)$ & $0.845(0.638-1.119)$ & 0.268 & \\
\hline IL12A rs568408 & $n=234$ & $n=240$ & & & \\
\hline GG & $173(0.74)$ & $171(0.71)$ & Referent & & 0.782 \\
\hline AG & $52(0.22)$ & $63(0.26)$ & $0.816(0.534-1.246)$ & 0.389 & \\
\hline AA & $9(0.04)$ & $6(0.03)$ & $1.483(0.517-4.256)$ & 0.600 & \\
\hline $\mathrm{AG}+\mathrm{AA}$ & $61(0.26)$ & $69(0.29)$ & $0.874(0.583-1.309)$ & 0.538 & \\
\hline MAF & $70(0.15)$ & $75(0.16)$ & $0.976(0.684-1.393)$ & 0.965 & \\
\hline IL12B rs3212227 & $n=247$ & $n=240$ & & & \\
\hline AA & $156(0.63)$ & $151(0.63)$ & Referent & & 0.639 \\
\hline $\mathrm{AC}$ & $84(0.34)$ & $77(0.32)$ & $1.056(0.721-1.547)$ & 0.846 & \\
\hline $\mathrm{CC}$ & $7(0.03)$ & $12(0.05)$ & $0.563(0.217-1.473)$ & 0.345 & \\
\hline $\mathrm{AC}+\mathrm{CC}$ & $91(0.37)$ & $89(0.37)$ & $0.990(0.685-1.430)$ & 1.000 & \\
\hline MAF & $98(0.20)$ & $101(0.21)$ & $0.927(0.680-1.268)$ & 0.699 & \\
\hline IL4R rs1805015 & $n=303$ & $n=225$ & & & \\
\hline TT & $205(0.68)$ & $162(0.72)$ & Referent & & 0.304 \\
\hline $\mathrm{CT}$ & $82(0.27)$ & $53(0.24)$ & $1.223(0.818-1.828)$ & 0.360 & \\
\hline $\mathrm{CC}$ & $16(0.05)$ & $10(0.04)$ & $1.264(0.559-2.861)$ & 0.684 & \\
\hline $\mathrm{CT}+\mathrm{CC}$ & $98(0.32)$ & $63(0.28)$ & $1.229(0.843-1.793)$ & 0.295 & \\
\hline MAF & $114(0.19)$ & $73(0.16)$ & $1.197(0.866-1.653)$ & 0.313 & \\
\hline IL13 rs20541 & $n=303$ & $n=230$ & & & \\
\hline $\mathrm{CC}$ & $168(0.55)$ & $124(0.54)$ & Referent & & 0.457 \\
\hline $\mathrm{CT}$ & $114(0.38)$ & $84(0.36)$ & $1.002(0.695-1.443)$ & 1.000 & \\
\hline $\mathrm{TT}$ & $21(0.07)$ & $22(0.10)$ & $0.705(0.371-1.338)$ & 0.324 & \\
\hline $\mathrm{CT}+\mathrm{TT}$ & $135(0.45)$ & $106(0.46)$ & $0.940(0.666-1.326)$ & 0.726 & \\
\hline MAF & $156(0.26)$ & $128(0.28)$ & $0.899(0.684-1.182)$ & 0.489 & \\
\hline IL28B rs8099917 & $n=339$ & $n=375$ & & & \\
\hline TT & $219(0.65)$ & $245(0.65)$ & Referent & & 0.504 \\
\hline GT & $107(0.31)$ & $123(0.33)$ & $0.973(0.709-1.336)$ & 0.872 & \\
\hline GG & $13(0.04)$ & $7(0.02)$ & $2.078(0.814-5.302)$ & 0.169 & \\
\hline $\mathrm{GT}+\mathrm{GG}$ & $120(0.35)$ & $130(0.35)$ & $1.033(0.759-1.405)$ & 0.875 & \\
\hline MAF & $133(0.20)$ & $137(0.18)$ & $1.092(0.837-1.423)$ & 0.560 & \\
\hline IL28B rs 12979860 & $n=336$ & $n=372$ & & & \\
\hline $\mathrm{CC}$ & $141(0.42)$ & $164(0.44)$ & Referent & & 0.669 \\
\hline $\mathrm{CT}$ & $157(0.47)$ & $166(0.45)$ & $1.100(0.804-1.505)$ & 0.576 & \\
\hline $\mathrm{TT}$ & $38(0.11)$ & $42(0.11)$ & $1.052(0.643-1.723)$ & 0.900 & \\
\hline $\mathrm{CT}+\mathrm{TT}$ & $195(0.56)$ & $208(0.56)$ & $1.090(0.809-1.469)$ & 0.595 & \\
\hline MAF & $116(0.29)$ & $250(0.34)$ & $1.049(0.842-1.307)$ & 0.713 & \\
\hline GC rs2298849 & $n=364^{\mathrm{a}}$ & $n=375$ & & & \\
\hline TT & $226(0.62)$ & $237(0.63)$ & Referent & & 0.250 \\
\hline $\mathrm{CT}$ & $110(0.30)$ & $124(0.33)$ & $0.930(0.679-1.274)$ & 0.688 & \\
\hline $\mathrm{CC}$ & $28(0.08)$ & $14(0.04)$ & $2.097(1.077-4.086)$ & 0.035 & \\
\hline $\mathrm{CT}+\mathrm{CC}$ & $138(0.38)$ & $138(0.37)$ & $1.049(0.778-1.413)$ & 0.762 & \\
\hline MAF & $166(0.23)$ & $152(0.20)$ & $1.162(0.907-1.490)$ & 0.262 & \\
\hline
\end{tabular}


TABLE 4: Continued.

\begin{tabular}{|c|c|c|c|c|c|}
\hline Parameter & $\begin{array}{c}\text { Type } 2 \text { DM } \\
\text { nephropathy } \\
\text { (frequency) }\end{array}$ & $\begin{array}{l}\text { Healthy subjects } \\
\text { (frequency) }\end{array}$ & Odds ratio $(95 \% \mathrm{CI})$ & Two-tailed $P$ & $P_{\text {trend }}$ \\
\hline$G C$ rs7041 & $n=343$ & $n=361$ & & & \\
\hline GG & $112(0.33)$ & $116(0.32)$ & Referent & & 0.572 \\
\hline GT & $163(0.47)$ & $186(0.52)$ & $0.908(0.650-1.268)$ & 0.609 & \\
\hline $\mathrm{TT}$ & $68(0.20)$ & $59(0.16)$ & $1.194(0.773-1.844)$ & 0.440 & \\
\hline $\mathrm{GT}+\mathrm{TT}$ & $231(0.67)$ & $245(0.68)$ & $0.977(0.712-1.339)$ & 0.936 & \\
\hline MAF & $299(0.44)$ & $304(0.42)$ & $1.062(0.860-1.312)$ & 0.612 & \\
\hline GC rs1155563 & $n=362$ & $n=377$ & & & \\
\hline $\mathrm{TT}$ & $180(0.50)$ & $189(0.50)$ & Referent & & 0.541 \\
\hline $\mathrm{CT}$ & $141(0.39)$ & $155(0.41)$ & $0.955(0.703-1.297)$ & 0.815 & \\
\hline $\mathrm{CC}$ & $41(0.11)$ & $33(0.09)$ & $1.305(0.789-2.155)$ & 0.311 & \\
\hline $\mathrm{CT}+\mathrm{CC}$ & $182(0.50)$ & $188(0.50)$ & $1.017(0.762-1.356)$ & 0.941 & \\
\hline MAF & $223(0.31)$ & $221(0.29)$ & $1.074(0.859-1.341)$ & 0.567 & \\
\hline VDR rs2228570 & $n=345$ & $n=371$ & & & \\
\hline $\mathrm{CC}$ & $101(0.29)$ & $103(0.28)$ & Referent & & 0.401 \\
\hline $\mathrm{CT}$ & $175(0.51)$ & $183(0.49)$ & $0.975(0.691-1.376)$ & 0.930 & \\
\hline $\mathrm{TT}$ & $69(0.20)$ & $85(0.23)$ & $0.828(0.544-1.260)$ & 0.394 & \\
\hline $\mathrm{CT}+\mathrm{TT}$ & $244(0.71)$ & $268(0.72)$ & $0.929(0.671-1.285)$ & 0.679 & \\
\hline MAF & $313(0.45)$ & $353(0.48)$ & $0.915(0.743-1.126)$ & 0.432 & \\
\hline VDR rs1544410 & $n=359$ & $n=372$ & & & \\
\hline GG & $137(0.38)$ & $148(0.40)$ & Referent & & 0.753 \\
\hline AG & $165(0.46)$ & $165(0.44)$ & $1.080(0.787-1.483)$ & 0.686 & \\
\hline $\mathrm{AA}$ & $57(0.16)$ & $59(0.16)$ & $1.044(0.678-1.607)$ & 0.912 & \\
\hline $\mathrm{AG}+\mathrm{AA}$ & $222(0.62)$ & $224(0.60)$ & $1.071(0.795-1.442)$ & 0.705 & \\
\hline MAF & $279(0.39)$ & $283(0.38)$ & $1.035(0.839-1.278)$ & 0.788 & \\
\hline RXRA rs10776909 & $n=364$ & $n=378$ & & & \\
\hline $\mathrm{CC}$ & $233(0.64)$ & $250(0.66)$ & Referent & & 0.426 \\
\hline $\mathrm{CT}$ & $111(0.30)$ & $112(0.30)$ & $1.063(0.774-1.461)$ & 0.746 & \\
\hline $\mathrm{TT}$ & $20(0.05)$ & $16(0.04)$ & $1.341(0.679-2.651)$ & 0.490 & \\
\hline $\mathrm{CT}+\mathrm{TT}$ & $131(0.36)$ & $128(0.34)$ & $1.098(0.812-1.485)$ & 0.590 & \\
\hline MAF & $151(0.21)$ & $144(0.19)$ & $1.112(0.862-1.435)$ & 0.452 & \\
\hline$R X R A$ rs10881578 & $n=365$ & $n=377$ & & & \\
\hline AA & $197(0.54)$ & $183(0.48)$ & Referent & & 0.168 \\
\hline AG & $134(0.37)$ & $154(0.41)$ & $0.808(0.775-1.046)$ & 0.185 & \\
\hline GG & $34(0.09)$ & $40(0.11)$ & $0.790(0.479-1.301)$ & 0.376 & \\
\hline $\mathrm{AG}+\mathrm{GG}$ & $168(0.46)$ & $194(0.51)$ & $0.804(0.603-1.073)$ & 0.143 & \\
\hline MAF & $202(0.28)$ & $234(0.31)$ & $0.850(0.680-1.063)$ & 0.172 & \\
\hline RXRA rs749759 & $n=355$ & $n=370$ & & & \\
\hline GG & $207(0.58)$ & $221(0.60)$ & Referent & & 0.850 \\
\hline AG & $125(0.35)$ & $123(0.33)$ & $1.085(0.794-1.216)$ & 0.632 & \\
\hline $\mathrm{AA}$ & $23(0.06)$ & $26(0.07)$ & $0.944(0.522-1.708)$ & 0.881 & \\
\hline $\mathrm{AG}+\mathrm{AA}$ & $148(0.42)$ & $149(0.40)$ & $1.061(0.789-1.426)$ & 0.706 & \\
\hline MAF & $171(0.24)$ & $175(0.24)$ & $1.024(0.804-1.304)$ & 0.894 & \\
\hline
\end{tabular}

ESRD: end-stage renal disease, DM: diabetes mellitus, and MAF: minor allele frequency.

${ }^{a}$ Not consistent with Hardy-Weinberg equilibrium.

rs12979860F 5'CGTGCCTGTCGTGTACTGAA3', and rs12979860R 5' AGGCTCAGGGTCAATCACAG3'.

Genotyping of the GC rs1155563, GC rs2298849, RXRA rs10881578, and RXRA rs10776909 polymorphisms was carried out by HRM on the Bio-Rad CFX96 Real Time PCR system (Bio-Rad, Hercules, CA). DNA fragments amplified with the use of specific primers were subjected to HRM with $0.1^{\circ} \mathrm{C}$ increments in temperatures ranging from 71 to $92^{\circ} \mathrm{C}$. Genotyping of the GC rs7041, RXRA rs749759, $V D R$ rs1544410, and $V D R$ rs 2228570 was performed using the polymerase chain reaction and restriction fragment length polymorphism (PCR-RFLP) method according to the 
TABLE 5: Comparison of the distribution of polymorphic variants of tested genes between ESRD patients treated with hemodialysis due to type 2 DM nephropathy and the most common causes of ESRD other than type 2 DM nephropathy (chronic glomerulonephritis, chronic tubulointerstitial nephritis, and hypertensive nephritis).

\begin{tabular}{|c|c|c|c|c|c|}
\hline Genotype & $\begin{array}{l}\text { Type } 2 \text { DM nephropathy } \\
\text { (frequency) }\end{array}$ & $\begin{array}{l}\text { Other causes of ESRD } \\
\text { (frequency) }\end{array}$ & Odds ratio $(95 \% \mathrm{CI})$ & Two-tailed $P$ & $P_{\text {trend }}$ \\
\hline IL18 rs360719 & $n=248$ & $n=353$ & & & \\
\hline TT & $133(0.54)$ & $186(0.53)$ & Referent & - & 0.362 \\
\hline $\mathrm{CT}$ & $102(0.41)$ & $135(0.38)$ & $1.057(0.752-1.485)$ & 0.795 & \\
\hline $\mathrm{CC}$ & $13(0.05)$ & $32(0.09)$ & $0.568(0.287-1.124)$ & 0.107 & \\
\hline $\mathrm{CT}+\mathrm{CC}$ & $115(0.46)$ & $167(0.47)$ & $0.963(0.696-1.334)$ & 0.868 & \\
\hline MAF & $128(0.26)$ & $199(0.28)$ & $0.886(0.684-1.149)$ & 0.370 & \\
\hline IL12A rs568408 & $n=234$ & $n=337$ & & & \\
\hline GG & $173(0.74)$ & $234(0.69)$ & Referent & - & 0.303 \\
\hline AG & $52(0.22)$ & $89(0.26)$ & $0.790(0.533-1.060)$ & 0.275 & \\
\hline AA & $9(0.04)$ & $14(0.04)$ & $0.870(0.368-2.055)$ & 0.831 & \\
\hline $\mathrm{AG}+\mathrm{AA}$ & $61(0.26)$ & $103(0.31)$ & $0.801(0.552-1.163)$ & 0.260 & \\
\hline MAF & $70(0.15)$ & $117(0.17)$ & $0.837(0.606-1.157)$ & 0.319 & \\
\hline IL12B rs3212227 & $n=247$ & $n=352$ & & & \\
\hline AA & $156(0.63)$ & $205(0.58)$ & Referent & - & 0.176 \\
\hline $\mathrm{AC}$ & $84(0.34)$ & $132(0.38)$ & $0.836(0.593-1.068)$ & 0.337 & \\
\hline CC & $7(0.03)$ & $15(0.04)$ & $0.613(0.244-1.540)$ & 0.376 & \\
\hline $\mathrm{AC}+\mathrm{CC}$ & $91(0.37)$ & $147(0.42)$ & $0.814(0.582-1.136)$ & 0.236 & \\
\hline MAF & $98(0.20)$ & $162(0.23)$ & $0.828(0.624-1.098)$ & 0.215 & \\
\hline$I L 4 R$ rs1805015 & $n=303$ & $n=436$ & & & \\
\hline TT & $205(0.68)$ & $295(0.68)$ & Referent & - & 0.871 \\
\hline $\mathrm{CT}$ & $82(0.27)$ & $121(0.28)$ & $0.975(0.700-2.360)$ & 0.933 & \\
\hline $\mathrm{CC}$ & $16(0.05)$ & $20(0.05)$ & $1.151(0.583-2.275)$ & 0.728 & \\
\hline $\mathrm{CT}+\mathrm{CC}$ & $98(0.32)$ & $141(0.32)$ & $1.000(0.731-1.368)$ & 1.000 & \\
\hline MAF & $114(0.19)$ & $161(0.18)$ & $1.023(0.784-1.335)$ & 0.919 & \\
\hline IL13 rs20541 & $n=303$ & $n=436$ & & & \\
\hline $\mathrm{CC}$ & $168(0.55)$ & $242(0.56)$ & Referent & - & 0.902 \\
\hline $\mathrm{CT}$ & $114(0.38)$ & $166(0.38)$ & $0.989(0.726-1.348)$ & 1.000 & \\
\hline $\mathrm{TT}$ & $21(0.07)$ & $28(0.06)$ & $1.080(0.594-1.967)$ & 0.878 & \\
\hline $\mathrm{CT}+\mathrm{TT}$ & $135(0.45)$ & $194(0.44)$ & $1.002(0.746-1.346)$ & 1.000 & \\
\hline MAF & $156(0.26)$ & $222(0.25)$ & $1.015(0.800-1.287)$ & 0.950 & \\
\hline IL28B rs8099917 & $n=339$ & $n=493$ & & & \\
\hline $\mathrm{TT}$ & $219(0.65)$ & $317(0.64)$ & Referent & - & 0.858 \\
\hline GT & $107(0.31)$ & $162(0.33)$ & $0.956(0.709-1.289)$ & 0.820 & \\
\hline GG & $13(0.04)$ & $14(0.03)$ & $1.344(0.620-2.916)$ & 0.549 & \\
\hline $\mathrm{GT}+\mathrm{GG}$ & $120(0.35)$ & $176(0.36)$ & $0.987(0.739-1.318)$ & 0.941 & \\
\hline MAF & $133(0.20)$ & $190(0.19)$ & $1.022(0.799-1.309)$ & 0.910 & \\
\hline IL28B rs12979860 & $n=336$ & $n=488$ & & & \\
\hline $\mathrm{CC}$ & $141(0.42)$ & $209(0.43)$ & Referent & - & 0.952 \\
\hline $\mathrm{CT}$ & $157(0.47)$ & $221(0.45)$ & $1.053(0.783-1.415)$ & 0.763 & \\
\hline $\mathrm{TT}$ & $38(0.11)$ & $58(0.12)$ & $0.971(0.612-1.541)$ & 0.907 & \\
\hline $\mathrm{CT}+\mathrm{TT}$ & $195(0.56)$ & $279(0.57)$ & $1.036(0.782-1.373)$ & 0.830 & \\
\hline MAF & $116(0.29)$ & $337(0.35)$ & $1.006(0.819-1.237)$ & 0.994 & \\
\hline GC rs2298849 & $n=364^{\mathrm{a}}$ & $n=524$ & & & \\
\hline TT & $226(0.62)$ & $339(0.65)$ & Referent & - & 0.109 \\
\hline $\mathrm{CT}$ & $110(0.30)$ & $165(0.31)$ & $1.000(0.745-1.342)$ & 1.000 & \\
\hline $\mathrm{CC}$ & $28(0.08)$ & $20(0.04)$ & $2.100(1.155-3.819)$ & 0.014 & \\
\hline $\mathrm{CT}+\mathrm{CC}$ & $138(0.38)$ & $185(0.35)$ & $1.119(0.848-1.477)$ & 0.436 & \\
\hline MAF & $166(0.23)$ & $205(0.20)$ & $1.215(0.964-1.530)$ & 0.111 & \\
\hline
\end{tabular}


TABLE 5: Continued.

\begin{tabular}{|c|c|c|c|c|c|}
\hline Genotype & $\begin{array}{c}\text { Type } 2 \text { DM nephropathy } \\
\text { (frequency) }\end{array}$ & $\begin{array}{c}\text { Other causes of ESRD } \\
\text { (frequency) }\end{array}$ & Odds ratio $(95 \% \mathrm{CI})$ & Two-tailed $P$ & $P_{\text {trend }}$ \\
\hline GC rs7041 & $n=343$ & $n=506$ & & & \\
\hline GG & $112(0.33)$ & $182(0.36)$ & Referent & - & 0.247 \\
\hline GT & $163(0.47)$ & $236(0.47)$ & $1.122(0.824-1.528)$ & 0.480 & \\
\hline $\mathrm{TT}$ & $68(0.20)$ & $88(0.17)$ & $1.256(0.846-1.863)$ & 0.267 & \\
\hline $\mathrm{GT}+\mathrm{TT}$ & $231(0.67)$ & $324(0.64)$ & $1.159(0.867-1.548)$ & 0.340 & \\
\hline MAF & $299(0.44)$ & $412(0.41)$ & $1.125(0.925-1.369)$ & 0.259 & \\
\hline GC rs1155563 & $n=362$ & $n=527$ & & & \\
\hline $\mathrm{TT}$ & $180(0.50)$ & $252(0.48)$ & Referent & - & 0.614 \\
\hline $\mathrm{CT}$ & $141(0.39)$ & $213(0.40)$ & $0.927(0.696-1.234)$ & 0.610 & \\
\hline $\mathrm{CC}$ & $41(0.11)$ & $62(0.12)$ & $0.926(0.597-1.435)$ & 0.740 & \\
\hline $\mathrm{CT}+\mathrm{CC}$ & $182(0.50)$ & $275(0.52)$ & $0.927(0.709-1.211)$ & 0.585 & \\
\hline MAF & $223(0.31)$ & $337(0.32)$ & $0.947(0.772-1.161)$ & 0.638 & \\
\hline VDR rs2228570 & $n=345$ & $n=503$ & & & \\
\hline CC & $101(0.29)$ & $130(0.26)$ & Referent & - & 0.541 \\
\hline $\mathrm{CT}$ & $175(0.51)$ & $275(0.55)$ & $0.819(0.594-1.130)$ & 0.249 & \\
\hline $\mathrm{TT}$ & $69(0.20)$ & $98(0.19)$ & $0.906(0.606-1.356)$ & 0.682 & \\
\hline $\mathrm{CT}+\mathrm{TT}$ & $244(0.71)$ & $373(0.74)$ & $0.842(0.620-1.143)$ & 0.273 & \\
\hline MAF & $313(0.45)$ & $471(0.47)$ & $0.943(0.776-1.145)$ & 0.588 & \\
\hline VDR rs1544410 & $n=359$ & $n=512$ & & & \\
\hline GG & $137(0.38)$ & $189(0.37)$ & Referent & - & 0.598 \\
\hline AG & $165(0.46)$ & $235(0.46)$ & $0.969(0.720-1.303)$ & 0.880 & \\
\hline AA & $57(0.16)$ & $88(0.17)$ & $0.894(0.599-1.332)$ & 0.613 & \\
\hline $\mathrm{AG}+\mathrm{AA}$ & $222(0.62)$ & $323(0.63)$ & $0.948(0.718-1.253)$ & 0.722 & \\
\hline MAF & $279(0.39)$ & $411(0.40)$ & $0.948(0.778-1.152)$ & 0.626 & \\
\hline RXRA rs10776909 & $n=364$ & $n=526$ & & & \\
\hline CC & $233(0.64)$ & $308(0.59)$ & Referent & - & 0.298 \\
\hline $\mathrm{CT}$ & $111(0.30)$ & $196(0.37)$ & $0.749(0.561-0.999)$ & 0.050 & \\
\hline $\mathrm{TT}$ & $20(0.05)$ & $22(0.04)$ & $1.202(0.641-2.254)$ & 0.629 & \\
\hline $\mathrm{CT}+\mathrm{TT}$ & $131(0.36)$ & $218(0.41)$ & $0.794(0.603-1.046)$ & 0.108 & \\
\hline MAF & $151(0.21)$ & $240(0.23)$ & $0.883(0.702-1.112)$ & 0.317 & \\
\hline RXRA rs10881578 & $n=365$ & $n=525$ & & & \\
\hline $\mathrm{AA}$ & $197(0.54)$ & $252(0.48)$ & Referent & - & 0.134 \\
\hline AG & $134(0.37)$ & $220(0.42)$ & $0.779(0.586-1.035)$ & 0.096 & \\
\hline GG & $34(0.09)$ & $53(0.10)$ & $0.821(0.513-1.312)$ & 0.478 & \\
\hline $\mathrm{AG}+\mathrm{GG}$ & $168(0.46)$ & $273(0.52)$ & $0.787(0.602-1.029)$ & 0.088 & \\
\hline MAF & $202(0.28)$ & $326(0.31)$ & $0.850(0.690-1.046)$ & 0.139 & \\
\hline RXRA rs749759 & $n=355$ & $n=514$ & & & \\
\hline GG & $207(0.58)$ & $265(0.52)$ & Referent & - & 0.082 \\
\hline AG & $125(0.35)$ & $212(0.41)$ & $0.755(0.567-1.005)$ & 0.059 & \\
\hline $\mathrm{AA}$ & $23(0.06)$ & $37(0.07)$ & $0.796(0.459-1.381)$ & 0.490 & \\
\hline $\mathrm{AG}+\mathrm{AA}$ & $148(0.42)$ & $249(0.48)$ & $0.761(0.579-1.000)$ & 0.053 & \\
\hline MAF & $171(0.24)$ & $286(0.28)$ & $0.823(0.661-1.025)$ & 0.092 & \\
\hline
\end{tabular}

ESRD: end-stage renal disease, DM: diabetes mellitus, and MAF: minor allele frequency.

${ }^{a}$ Not consistent with Hardy-Weinberg equilibrium.

manufacturer's instructions (Fermentas, Vilnius, Lithuania). Primer sequences and conditions for HRM and PCR-RFLP analyses are presented in Table 1.

For quality control, the genotyping analysis was blinded to the subject's case-control status. In addition, approximately $10 \%$ of the randomly chosen samples were regenotyped. Samples that failed the genotyping were excluded from further statistical analyses.
2.3. $25(\mathrm{OH}) \mathrm{D}$ Testing. Plasma $25(\mathrm{OH}) \mathrm{D}$ was determined in blindly selected $162 \mathrm{HD}$ patients in the winter season of the year to avoid differences in sunlight exposure between patients who used to sunbathe and those who did not. Plasma $25(\mathrm{OH}) \mathrm{D}$ concentration was measured in HD patients who had not been treated with vitamin D or had stopped such a treatment for at least 3 weeks to obtain the so-called basic vitamin D concentrations. Under these conditions, there were 
TABLE 6: Selected comparisons of the polymorphic variants distribution of tested genes between type 2 DM nephropathy patients, chronic infective tubulointerstitial nephritic patients, and healthy subjects.

\begin{tabular}{|c|c|c|c|c|c|}
\hline \multirow{2}{*}{\multicolumn{6}{|c|}{$\begin{array}{l}\text { Genotype frequencies } \\
\text { Type } 2 \text { DM nephropathy versus chronic infective tubulointerstitial nephritis }\end{array}$}} \\
\hline & & & & & \\
\hline IL18 rs360719 & $n=248$ & \multicolumn{3}{|l|}{$n=77$} & \\
\hline TT & $133(0.54)$ & $54(0.70)$ & \multicolumn{2}{|l|}{ Referent } & \multirow[t]{5}{*}{0.033} \\
\hline $\mathrm{CT}$ & $102(0.41)$ & $19(0.25)$ & $2.180(1.217-3.905)$ & $0.009^{\mathrm{a}}$ & \\
\hline $\mathrm{CC}$ & $13(0.05)$ & $4(0.05)$ & $1.320(0.412-4.228)$ & 0.783 & \\
\hline $\mathrm{CT}+\mathrm{CC}$ & $115(0.46)$ & $23(0.30)$ & $2.030(1.173-3.512)$ & $0.012^{\mathrm{a}}$ & \\
\hline MAF & $128(0.26)$ & $27(0.18)$ & $1.636(1.031-2.596)$ & 0.046 & \\
\hline \multicolumn{6}{|c|}{ Chronic infective tubulointerstitial nephritis versus healthy controls } \\
\hline IL18 rs360719 & $n=77$ & $n=240$ & & & \\
\hline $\mathrm{TT}$ & $54(0.70)$ & $121(0.50)$ & Referent & & 0.005 \\
\hline $\mathrm{CT}$ & $19(0.25)$ & $98(0.41)$ & $0.434(0.242-0.781)$ & $0.006^{\mathrm{a}}$ & \\
\hline CC & $4(0.05)$ & $21(0.09)$ & $0.427(0.140-1.303)$ & 0.160 & \\
\hline $\mathrm{CT}+\mathrm{CC}$ & $23(0.30)$ & $119(0.50)$ & $0.433(0.250-0.750)$ & $0.004^{\mathrm{a}}$ & \\
\hline MAF & $27(0.18)$ & $140(0.29)$ & $0.516(0.326-0.818)$ & 0.006 & \\
\hline
\end{tabular}

DM: diabetes mellitus; MAF: minor allele frequency.

Significant differences are indicated using bold font.

${ }^{a}$ Significant after the Bonferroni correction $(P<0.017)$.

no patients showing optimal plasma 25(OH)D levels (35$80 \mathrm{ng} / \mathrm{mL}$ for adults). To examine plasma $25(\mathrm{OH}) \mathrm{D}$ levels, a chemiluminescent microparticle immunoassay (CMIA) was used according to the manufacturer's instructions (Abbott Diagnostics ARCHITECT 25-OH VITAMIN D CMIA).

2.4. Statistical Methods. Results are presented as percentage for categorical variables, as mean with one standard deviation for normally distributed continuous variables or as median with range for not normally distributed continuous variables as tested by the Shapiro-Wilk test. Statistical tests used for comparison of data obtained in selected groups are indicated at $P$ values.

Hardy-Weinberg equilibrium (HWE) was tested to compare the observed genotype frequencies to the expected ones using Chi-square test. Distributions of tested polymorphisms were consistent with HWE with three exceptions which are indicated in tables showing analysis of genotype and allele distributions. The Fisher exact probability test or Chi-square test was used to evaluate differences in genotype and allele prevalence between the examined groups. Homozygotes for the major allele were the reference group. The odds ratio (OR) with $P$ value and 95\% confidence intervals $(95 \%$ $\mathrm{CI})$ value were calculated. All probabilities were two-tailed. Polymorphisms were tested for association using the Chisquare test for trend $\left(P_{\text {trend }}\right)$. Power analysis was performed by Fisher's exact test.

Values of $P<0.05$ were judged to be significant. However, associations were reported only if the following conditions were fulfilled.

(1) A genotype distribution was consistent with HWE in a tested group and a referent group.

(2) $P_{\text {trend }}$ was below 0.05 .
(3) Odds ratio remained significant after the Bonferroni correction applied for multiple testing, if appropriate.

Aforementioned statistical calculations were performed using GraphPad InStat 3.10, 32 bit for Windows, created on July 9, 2009 (GraphPad Software, Inc., La Jolla, USA), CytelStudio version 10.0, created on January 16, 2013 (CytelStudio Software Corporation, Cambridge, USA), and Statistica version 10, 2011 (StatSoft, Inc., Tulsa, USA).

\section{Results}

Characteristics of the examined HD patients are presented in Tables 2 and 3. ESRD patients due to type 2 DM nephropathy compared to non-DM ESRD patients showed older age at RRT onset, shorter treatment with RRT, higher death rate on RRT, higher prevalence of CAD and myocardial infarction, lower serum PTH level, and lower frequency of parathyroidectomy and treatment with cinacalcet.

In respect of the examined parameters, type $2 \mathrm{DM}$ nephropathy patients differed the most significantly from chronic glomerulonephritic subjects, the least significantly from hypertensive nephropathy patients.

There were no differences in frequency distributions of tested genotypes between type 2 DM nephropathy patients and healthy subjects (Table 4) as well as other ESRD patients analyzed together (Table 5) which could be judged as significant associations.

Comparisons of genotype and allele frequencies between type 2 DM nephropathy patients and other ESRD groups revealed associations only with chronic infective tubulointerstitial nephritic patients in respect of IL18 rs360719 (Table 6, no significant results are shown). Frequency of IL18 rs360719 allele C carriers was higher in type 2 DM nephropathy patients than in those with chronic infective tubulointerstitial 
TABLE 7: Comparison of the distribution of polymorphic variants of tested genes between ESRD patients treated with hemodialysis due to type 2 DM nephropathy grouped by diagnosis of CAD.

\begin{tabular}{|c|c|c|c|c|c|}
\hline Parameter & $\begin{array}{l}\text { Type } 2 \text { DM nephropathy } \\
\text { with CAD (frequency) }\end{array}$ & $\begin{array}{c}\text { Type } 2 \mathrm{DM} \\
\text { nephropathy without } \\
\text { CAD (frequency) }\end{array}$ & Odds ratio $(95 \% \mathrm{CI})$ & Two-tailed $P$ & $P_{\text {trend }}$ \\
\hline IL18 rs360719 & $n=124$ & $n=109$ & & & \\
\hline TT & $68(0.55)$ & $53(0.49)$ & Referent & & 0.269 \\
\hline $\mathrm{CT}$ & $51(0.41)$ & $49(0.45)$ & $1.128(0.725-1.754)$ & 0.653 & \\
\hline $\mathrm{CC}$ & $5(0.04)$ & $7(0.06)$ & $0.628(0.194-2.036)$ & 0.557 & \\
\hline $\mathrm{CT}+\mathrm{CC}$ & $56(0.45)$ & $56(0.51)$ & $0.879(0.560-1.380)$ & 0.645 & \\
\hline MAF & $61(0.25)$ & $63(0.29)$ & $0.803(0.532-1.211)$ & 0.345 & \\
\hline IL12A rs568408 & $n=117$ & $n=102$ & & & \\
\hline GG & $83(0.71)$ & $77(0.63)$ & Referent & & 0.361 \\
\hline AG & $28(0.24)$ & $22(0.22)$ & $1.181(0.623-2.236)$ & 0.630 & \\
\hline $\mathrm{AA}$ & $6(0.05)$ & $3(0.03)$ & $1.855(0.448-7.678)$ & 0.502 & \\
\hline $\mathrm{AG}+\mathrm{AA}$ & $34(0.29)$ & $25(0.25)$ & $1.262(0.691-2.304)$ & 0.542 & \\
\hline MAF & $40(0.17)$ & $28(0.14)$ & $1.311(0.776-2.214)$ & 0.378 & \\
\hline IL12B rs3212227 & $n=124$ & $n=109$ & & & \\
\hline AA & $78(0.63)$ & $69(0.63)$ & Referent & & 0.906 \\
\hline $\mathrm{AC}$ & $43(0.35)$ & $36(0.33)$ & $1.057(0.611-1.829)$ & 0.889 & \\
\hline $\mathrm{CC}$ & $3(0.02)$ & $4(0.04)$ & $0.664(0.143-3.069)$ & 0.708 & \\
\hline $\mathrm{AC}+\mathrm{CC}$ & $46(0.37)$ & $40(0.37)$ & $1.017(0.597-1.734)$ & 1.000 & \\
\hline MAF & $49(0.20)$ & $44(0.20)$ & $0.974(0.618-1.535)$ & 0.909 & \\
\hline IL4R rs1805015 & $n=144$ & $n=127$ & & & \\
\hline $\mathrm{TT}$ & $95(0.66)$ & $86(0.68)$ & Referent & & 0.947 \\
\hline $\mathrm{CT}$ & $42(0.29)$ & $32(0.25)$ & $1.188(0.689-2.048)$ & 0.581 & \\
\hline $\mathrm{CC}$ & $7(0.05)$ & $9(0.07)$ & $0.704(0.251-1.972)$ & 0.605 & \\
\hline $\mathrm{CT}+\mathrm{CC}$ & $49(0.34)$ & $41(0.32)$ & $1.082(0.652-1.797)$ & 0.797 & \\
\hline MAF & $56(0.19)$ & $50(0.20)$ & $0.985(0.644-1.504)$ & 0.944 & \\
\hline IL13 rs20541 & $n=144$ & $n=127$ & & & \\
\hline $\mathrm{CC}$ & $80(0.56)$ & $71(0.56)$ & Referent & & 0.867 \\
\hline $\mathrm{CT}$ & $55(0.38)$ & $46(0.36)$ & $1.061(0.640-1.759)$ & 0.898 & \\
\hline $\mathrm{TT}$ & $9(0.06)$ & $10(0.08)$ & $0.799(0.307-2.077)$ & 0.808 & \\
\hline $\mathrm{CT}+\mathrm{TT}$ & $64(0.44)$ & $56(0.44)$ & $1.014(0.627-1.640)$ & 1.000 & \\
\hline MAF & $73(0.25)$ & $92(0.26)$ & $0.967(0.657-1.423)$ & 0.944 & \\
\hline IL28B rs8099917 & $n=163$ & $n=145$ & & & \\
\hline TT & $105(0.64)$ & $97(0.67)$ & Referent & & 0.752 \\
\hline GT & $52(0.32)$ & $42(0.29)$ & $1.144(0.700-1.870)$ & 0.618 & \\
\hline GG & $6(0.04)$ & $6(0.04)$ & $0.924(0.288-2.961)$ & 1.000 & \\
\hline $\mathrm{GT}+\mathrm{GG}$ & $58(0.36)$ & $48(0.33)$ & $1.116(0.697-1.189)$ & 0.719 & \\
\hline MAF & $64(0.20)$ & $54(0.19)$ & $1.068(0.714-1.597)$ & 0.829 & \\
\hline IL28B rs12979860 & $n=163$ & $n=142$ & & & \\
\hline $\mathrm{CC}$ & $69(0.42)$ & $66(0.46)$ & Referent & & 0.352 \\
\hline $\mathrm{CT}$ & $73(0.45)$ & $62(0.44)$ & $1.126(0.698-1.816)$ & 0.715 & \\
\hline $\mathrm{TT}$ & $21(0.13)$ & $14(0.10)$ & $1.435(0.674-3.055)$ & 0.448 & \\
\hline $\mathrm{CT}+\mathrm{TT}$ & $94(0.58)$ & $76(0.54)$ & $1.183(0.752-1.861)$ & 0.490 & \\
\hline MAF & $115(0.35)$ & $90(0.32)$ & $1.175(0.838-1.647)$ & 0.396 & \\
\hline GC rs2298849 & $n=172$ & $n=158^{\mathrm{a}}$ & & & \\
\hline TT & $99(0.58)$ & $106(0.67)$ & Referent & & 0.173 \\
\hline $\mathrm{CT}$ & $60(0.35)$ & $40(0.25)$ & $1.606(0.989-2.608)$ & 0.067 & \\
\hline $\mathrm{CC}$ & $13(0.07)$ & $12(0.08)$ & $1.160(0.505-2.663)$ & 0.833 & \\
\hline $\mathrm{CT}+\mathrm{CC}$ & $73(0.42)$ & $52(0.33)$ & $1.503(0.959-2.355)$ & 0.088 & \\
\hline MAF & $166(0.25)$ & $64(0.20)$ & $1.313(0.909-1.895)$ & 0.174 & \\
\hline
\end{tabular}


TABLE 7: Continued.

\begin{tabular}{|c|c|c|c|c|c|}
\hline Parameter & $\begin{array}{l}\text { Type } 2 \text { DM nephropathy } \\
\text { with CAD (frequency) }\end{array}$ & $\begin{array}{c}\text { Type } 2 \mathrm{DM} \\
\text { nephropathy without } \\
\text { CAD (frequency) }\end{array}$ & Odds ratio $(95 \% \mathrm{CI})$ & Two-tailed $P$ & $P_{\text {trend }}$ \\
\hline GC rs7041 & $n=161$ & $n=151$ & & & \\
\hline GG & $57(0.35)$ & $46(0.30)$ & Referent & & 0.844 \\
\hline GT & $69(0.43)$ & $82(0.54)$ & $1.327(0.825-2.134)$ & 0.277 & \\
\hline $\mathrm{TT}$ & $35(0.22)$ & $23(0.15)$ & $1.629(0.900-2.949)$ & 0.136 & \\
\hline $\mathrm{GT}+\mathrm{TT}$ & $104(0.65)$ & $105(0.70)$ & $1.061(0.721-1.559)$ & 0.769 & \\
\hline MAF & $139(0.43)$ & $128(0.42)$ & $1.025(0.746-1.409)$ & 0.943 & \\
\hline GC rs1155563 & $n=172$ & $n=157$ & & & \\
\hline TT & $82(0.48)$ & $79(0.50)$ & Referent & & 0.645 \\
\hline $\mathrm{CT}$ & $70(0.41)$ & $61(0.39)$ & $1.106(0.697-1.755)$ & 0.724 & \\
\hline $\mathrm{CC}$ & $20(0.12)$ & $17(0.11)$ & $1.133(0.554-2.321)$ & 0.856 & \\
\hline $\mathrm{CT}+\mathrm{CC}$ & $90(0.52)$ & $78(0.50)$ & $1.112(0.721-1.714)$ & 0.660 & \\
\hline MAF & $110(0.32)$ & $95(0.30)$ & $1.084(0.779-1.508)$ & 0.695 & \\
\hline VDR rs 2228570 & $n=162$ & $n=152$ & & & \\
\hline $\mathrm{CC}$ & $43(0.27)$ & $44(0.29)$ & Referent & & 0.316 \\
\hline $\mathrm{CT}$ & $93(0.57)$ & $68(0.45)$ & $1.400(0.829-2.363)$ & 0.230 & \\
\hline $\mathrm{TT}$ & $26(0.16)$ & $40(0.26)$ & $0.665(0.348-1.272)$ & 0.252 & \\
\hline $\mathrm{CT}+\mathrm{TT}$ & $119(0.73)$ & $108(0.71)$ & $1.128(0.688-1.849)$ & 0.705 & \\
\hline MAF & $145(0.45)$ & $148(0.49)$ & $0.854(0.624-1.169)$ & 0.365 & \\
\hline VDR rs1544410 & $n=170$ & $n=155$ & & & \\
\hline GG & $65(0.38)$ & $61(0.39)$ & Referent & & 0.772 \\
\hline AG & $79(0.46)$ & $72(0.46)$ & $1.030(0.641-1.653)$ & 0.905 & \\
\hline AA & $26(0.15)$ & $22(0.14)$ & $1.109(0.569-2.160)$ & 0.865 & \\
\hline $\mathrm{AG}+\mathrm{AA}$ & $105(0.62)$ & $94(0.61)$ & $1.048(0.671-1.639)$ & 0.909 & \\
\hline MAF & $131(0.39)$ & $116(0.37)$ & $1.048(0.763-1.440)$ & 0.833 & \\
\hline$R X R A$ rs 10776909 & $n=172$ & $n=158$ & & & \\
\hline $\mathrm{CC}$ & $112(0.65)$ & $104(0.66)$ & Referent & & 0.621 \\
\hline $\mathrm{CT}$ & $48(0.28)$ & $47(0.30)$ & $0.948(0.585-1.537)$ & 0.902 & \\
\hline $\mathrm{TT}$ & $12(0.07)$ & $7(0.04)$ & $1.592(0.604-4.198)$ & 0.473 & \\
\hline $\mathrm{CT}+\mathrm{TT}$ & $60(0.35)$ & $54(0.34)$ & $1.032(0.655-1.625)$ & 0.908 & \\
\hline MAF & $72(0.21)$ & $61(0.19)$ & $1.107(0.756-1.621)$ & 0.672 & \\
\hline RXRA rs10881578 & $n=173$ & $n=158$ & & & \\
\hline AA & $89(0.51)$ & $92(0.58)$ & Referent & & 0.192 \\
\hline AG & $65(0.38)$ & $53(0.34)$ & $1.268(0.796-2.019)$ & 0.345 & \\
\hline GG & $19(0.11)$ & $13(0.08)$ & $1.511(0.704-3.241)$ & 0.340 & \\
\hline $\mathrm{AG}+\mathrm{GG}$ & $84(0.49)$ & $66(0.42)$ & $1.316(0.852-2.032)$ & 0.226 & \\
\hline MAF & $103(0.30)$ & $79(0.25)$ & $1.272(0.902-1.793)$ & 0.199 & \\
\hline RXRA rs 749759 & $n=169$ & $n=153$ & & & \\
\hline GG & $100(0.59)$ & $89(0.58)$ & Referent & & 0.812 \\
\hline AG & $59(0.35)$ & $54(0.35)$ & $0.972(0.610-1.551)$ & 1.000 & \\
\hline $\mathrm{AA}$ & $10(0.06)$ & $10(0.07)$ & $0.890(0.354-2.238)$ & 0.818 & \\
\hline $\mathrm{AG}+\mathrm{AA}$ & $69(0.41)$ & $64(0.42)$ & $0.960(0.615-1.496)$ & 0.910 & \\
\hline MAF & $79(0.23)$ & $74(0.24)$ & $0.956(0.665-1.375)$ & 0.882 & \\
\hline
\end{tabular}

CAD: coronary artery disease, ESRD: end-stage renal disease, DM: diabetes mellitus, and MAF: minor allele frequency.

${ }^{a}$ Not consistent with Hardy-Weinberg equilibrium.

nephritis. The latter group showed lower frequency of IL18 rs360719 allele C carriers compared to healthy controls (Table 6).

Type 2 DM nephropathy patients with diagnosed CAD differed in tested genotype frequencies neither from type 2 DM nephropathy subjects without CAD (Table 7) nor from healthy controls (Table 8).

\section{Discussion}

Genetic studies involving DM nephropathy and related complications are not consistent in many aspects [31-34]. Some polymorphisms tested in this study were reported as being associated with type 1 DM (IL12B rs3212227 [35], IL4R [36, 37], IL13 [37], VDR rs1544410 [38, 39], and VDR 
TABLE 8: Comparison of the distribution of polymorphic variants of tested genes between type 2 DM nephropathy patients with diagnosis of CAD and healthy controls.

\begin{tabular}{|c|c|c|c|c|c|}
\hline Parameter & $\begin{array}{l}\text { Type } 2 \text { DM nephropathy } \\
\text { with CAD (frequency) }\end{array}$ & $\begin{array}{l}\text { Healthy controls } \\
\text { (frequency) }\end{array}$ & Odds ratio $(95 \% \mathrm{CI})$ & Two-tailed $P$ & $P_{\text {trend }}$ \\
\hline IL18 rs360719 & $n=124$ & $n=240$ & & & \\
\hline TT & $68(0.55)$ & $121(0.50)$ & Referent & & 0.186 \\
\hline CT & $51(0.41)$ & $98(0.41)$ & $0.926(0.590-1.453)$ & 0.819 & \\
\hline $\mathrm{CC}$ & $5(0.04)$ & $21(0.09)$ & $0.424(0.153-1.174)$ & 0.122 & \\
\hline $\mathrm{CT}+\mathrm{CC}$ & $56(0.45)$ & $119(0.50)$ & $0.837(0.542-1.294)$ & 0.440 & \\
\hline MAF & $61(0.25)$ & $140(0.29)$ & $0.792(0.558-1.124)$ & 0.223 & \\
\hline IL12A rs568408 & $n=117$ & $n=240$ & & & \\
\hline GG & $83(0.71)$ & $171(0.71)$ & Referent & & 0.626 \\
\hline AG & $28(0.24)$ & $63(0.26)$ & $0.916(0.546-1.535)$ & 0.794 & \\
\hline $\mathrm{AA}$ & $6(0.05)$ & $6(0.03)$ & $2.060(0.645-6.583)$ & 0.348 & \\
\hline $\mathrm{AG}+\mathrm{AA}$ & $34(0.29)$ & $69(0.29)$ & $1.015(0.624-1.653)$ & 1.000 & \\
\hline MAF & $40(0.17)$ & $75(0.16)$ & $1.113(0.731-1.695)$ & 0.695 & \\
\hline IL12B rs3212227 & $n=124$ & $n=240$ & & & \\
\hline AA & $78(0.63)$ & $151(0.63)$ & Referent & & 0.475 \\
\hline $\mathrm{AC}$ & $43(0.35)$ & $77(0.32)$ & $1.081(0.681-1.717)$ & 0.813 & \\
\hline $\mathrm{CC}$ & $3(0.02)$ & $12(0.05)$ & $0.484(0.133-1.766)$ & 0.397 & \\
\hline $\mathrm{AC}+\mathrm{CC}$ & $46(0.37)$ & $89(0.37)$ & $1.001(0.639-1.567)$ & 1.000 & \\
\hline MAF & $49(0.20)$ & $101(0.21)$ & $0.924(0.631-1.354)$ & 0.757 & \\
\hline IL4R rs1805015 & $n=144$ & $n=225$ & & & \\
\hline TT & $95(0.66)$ & $162(0.72)$ & Referent & & 0.285 \\
\hline $\mathrm{CT}$ & $42(0.29)$ & $53(0.24)$ & $1.351(0.838-2.179)$ & 0.221 & \\
\hline $\mathrm{CC}$ & $7(0.05)$ & $10(0.04)$ & $1.194(0.440-3.240)$ & 0.798 & \\
\hline $\mathrm{CT}+\mathrm{CC}$ & $49(0.34)$ & $63(0.28)$ & $1.326(0.845-2.083)$ & 0.246 & \\
\hline MAF & $56(0.19)$ & $73(0.16)$ & $1.247(0.848-1.832)$ & 0.305 & \\
\hline IL13 rs20541 & $n=144$ & $n=230$ & & & \\
\hline $\mathrm{CC}$ & $80(0.56)$ & $124(0.54)$ & Referent & & 0.469 \\
\hline $\mathrm{CT}$ & $55(0.38)$ & $84(0.36)$ & $1.015(0.653-1.578)$ & 1.000 & \\
\hline $\mathrm{TT}$ & $9(0.06)$ & $22(0.10)$ & $0.634(0.278-1.447)$ & 0.324 & \\
\hline $\mathrm{CT}+\mathrm{TT}$ & $64(0.44)$ & $106(0.46)$ & $0.936(0.616-1.422)$ & 0.831 & \\
\hline MAF & $73(0.25)$ & $128(0.28)$ & $0.881(0.630-1.231)$ & 0.510 & \\
\hline IL28B rs8099917 & $n=163$ & $n=375$ & & & \\
\hline $\mathrm{TT}$ & $105(0.64)$ & $245(0.65)$ & Referent & & 0.584 \\
\hline GT & $52(0.32)$ & $123(0.33)$ & $0.986(0.663-1.467)$ & 1.000 & \\
\hline GG & $6(0.04)$ & $7(0.02)$ & $2.000(0.656-6.094)$ & 0.229 & \\
\hline $\mathrm{GT}+\mathrm{GG}$ & $58(0.36)$ & $130(0.35)$ & $1.041(0.709-1.530)$ & 0.845 & \\
\hline MAF & $64(0.20)$ & $137(0.18)$ & $1.093(0.786-1.521)$ & 0.658 & \\
\hline IL28B rs12979860 & $n=163$ & $n=372$ & & & \\
\hline $\mathrm{CC}$ & $69(0.42)$ & $164(0.44)$ & Referent & & 0.281 \\
\hline $\mathrm{CT}$ & $73(0.45)$ & $166(0.45)$ & $1.045(0.705-1.549)$ & 0.841 & \\
\hline $\mathrm{TT}$ & $21(0.13)$ & $42(0.11)$ & $1.188(0.656-2.154)$ & 0.644 & \\
\hline $\mathrm{CT}+\mathrm{TT}$ & $94(0.58)$ & $208(0.56)$ & $1.074(0.740-1.558)$ & 0.776 & \\
\hline MAF & $115(0.35)$ & $250(0.34)$ & $1.077(0.819-1.416)$ & 0.644 & \\
\hline GC rs2298849 & $n=172$ & $n=375$ & & & \\
\hline TT & $99(0.58)$ & $237(0.63)$ & Referent & & 0.080 \\
\hline $\mathrm{CT}$ & $60(0.35)$ & $124(0.33)$ & $1.158(0.786-1.706)$ & 0.486 & \\
\hline $\mathrm{CC}$ & $13(0.07)$ & $14(0.04)$ & $2.223(1.008-4.901)$ & 0.052 & \\
\hline $\mathrm{CT}+\mathrm{CC}$ & $73(0.42)$ & $138(0.37)$ & $1.266(0.876-1.830)$ & 0.220 & \\
\hline MAF & $166(0.25)$ & $152(0.20)$ & $1.311(0.969-1.774)$ & 0.092 & \\
\hline
\end{tabular}


TABLE 8: Continued.

\begin{tabular}{|c|c|c|c|c|c|}
\hline Parameter & $\begin{array}{l}\text { Type } 2 \text { DM nephropathy } \\
\text { with CAD (frequency) }\end{array}$ & $\begin{array}{c}\text { Healthy controls } \\
\text { (frequency) }\end{array}$ & Odds ratio $(95 \% \mathrm{CI})$ & Two-tailed $P$ & $P_{\text {trend }}$ \\
\hline GC rs7041 & $n=161$ & $n=361$ & & & \\
\hline GG & $57(0.35)$ & $116(0.32)$ & Referent & & 0.748 \\
\hline GT & $69(0.43)$ & $186(0.52)$ & $0.755(0.496-1.150)$ & 0.196 & \\
\hline TT & $35(0.22)$ & $59(0.16)$ & $1.207(0.714-2.040)$ & 0.502 & \\
\hline $\mathrm{GT}+\mathrm{TT}$ & $104(0.65)$ & $245(0.68)$ & $0.864(0.584-1.278)$ & 0.482 & \\
\hline MAF & $139(0.43)$ & $304(0.42)$ & $1.044(0.801-1.362)$ & 0.800 & \\
\hline GC rs1155563 & $n=172$ & $n=377$ & & & \\
\hline $\mathrm{TT}$ & $82(0.48)$ & $189(0.50)$ & Referent & & 0.378 \\
\hline $\mathrm{CT}$ & $70(0.41)$ & $155(0.41)$ & $1.041(0.710-1.527)$ & 0.845 & \\
\hline $\mathrm{CC}$ & $20(0.12)$ & $33(0.09)$ & $1.397(0.757-2.578)$ & 0.332 & \\
\hline $\mathrm{CT}+\mathrm{CC}$ & $90(0.52)$ & $188(0.50)$ & $1.103(0.769-1.583)$ & 0.646 & \\
\hline MAF & $110(0.32)$ & $221(0.29)$ & $1.134(0.861-1.494)$ & 0.411 & \\
\hline$V D R$ rs2228570 & $n=162$ & $n=371$ & & & \\
\hline $\mathrm{CC}$ & $43(0.27)$ & $103(0.28)$ & Referent & & 0.386 \\
\hline CT & $93(0.57)$ & $183(0.49)$ & $1.217(0.788-1.880)$ & 0.384 & \\
\hline $\mathrm{TT}$ & $26(0.16)$ & $85(0.23)$ & $0.733(0.416-1.290)$ & 0.321 & \\
\hline $\mathrm{CT}+\mathrm{TT}$ & $119(0.73)$ & $268(0.72)$ & $1.064(0.702-1.613)$ & 0.833 & \\
\hline MAF & $145(0.45)$ & $353(0.48)$ & $0.893(0.687-1.160)$ & 0.434 & \\
\hline$V D R$ rs1544410 & $n=170$ & $n=372$ & & & \\
\hline GG & $65(0.38)$ & $148(0.40)$ & Referent & & 0.880 \\
\hline AG & $79(0.46)$ & $165(0.44)$ & $1.090(0.734-1.620)$ & 0.687 & \\
\hline AA & $26(0.15)$ & $59(0.16)$ & $1.003(0.581-1.732)$ & 1.000 & \\
\hline $\mathrm{AG}+\mathrm{AA}$ & $105(0.62)$ & $224(0.60)$ & $1.067(0.735-1.549)$ & 0.776 & \\
\hline MAF & $131(0.39)$ & $283(0.38)$ & $1.021(0.784-1.329)$ & 0.931 & \\
\hline$R X R A$ rs10776909 & $n=172$ & $n=378$ & & & \\
\hline $\mathrm{CC}$ & $112(0.65)$ & $250(0.66)$ & Referent & & 0.483 \\
\hline $\mathrm{CT}$ & $48(0.28)$ & $112(0.30)$ & $0.957(0.638-1.434)$ & 0.838 & \\
\hline $\mathrm{TT}$ & $12(0.07)$ & $16(0.04)$ & $1.674(0.767-3.656)$ & 0.209 & \\
\hline $\mathrm{CT}+\mathrm{TT}$ & $60(0.35)$ & $128(0.34)$ & $1.046(0.716-1.529)$ & 0.846 & \\
\hline MAF & $72(0.21)$ & $144(0.19)$ & $1.125(0.819-1.545)$ & 0.518 & \\
\hline RXRA rs10881578 & $n=173$ & $n=377$ & & & \\
\hline AA & $89(0.51)$ & $183(0.48)$ & Referent & & 0.682 \\
\hline AG & $65(0.38)$ & $154(0.41)$ & $0.868(0.591-1.275)$ & 0.494 & \\
\hline GG & $19(0.11)$ & $40(0.11)$ & $0.977(0.535-1.783)$ & 1.000 & \\
\hline $\mathrm{AG}+\mathrm{GG}$ & $84(0.49)$ & $194(0.51)$ & $0.890(0.621-1.276)$ & 0.582 & \\
\hline MAF & $103(0.30)$ & $234(0.31)$ & $0.942(0.714-1.243)$ & 0.725 & \\
\hline RXRA rs749759 & $n=169$ & $n=370$ & & & \\
\hline GG & $100(0.59)$ & $221(0.60)$ & Referent & & 0.924 \\
\hline AG & $59(0.35)$ & $123(0.33)$ & $1.060(0.718-1.566)$ & 0.842 & \\
\hline $\mathrm{AA}$ & $10(0.06)$ & $26(0.07)$ & $0.850(0.395-1.830)$ & 0.710 & \\
\hline $\mathrm{AG}+\mathrm{AA}$ & $69(0.41)$ & $149(0.40)$ & $1.023(0.707-1.482)$ & 0.925 & \\
\hline MAF & $79(0.23)$ & $175(0.24)$ & $0.985(0.727-1.334)$ & 0.983 & \\
\hline
\end{tabular}

CAD: coronary artery disease, DM: diabetes mellitus, and MAF: minor allele frequency. 
rs2228570 [38]), type 2 DM susceptibility (VDR rs2228570 [40], VDR rs1544410 [41]), and phenotype of type $2 \mathrm{DM}$ (VDR rs2228570 [42], VDR rs1544410 [41, 43]). VDR rs2228570 and IL4 polymorphisms were also related to the risk of chronic kidney disease $[44,45]$. On the other hand, there are also data indicating no major effect of IL12B on type $1 \mathrm{DM}$ susceptibility in the entire study group [46], no association of $I L 4 R$ with type $1 \mathrm{DM}$ [47], no evident causal relationship between vitamin D pathway genes and type $2 \mathrm{DM}$, myocardial infarction or mortality [48], similar distribution of genotypes, allele and haplotypes of VDR rs2228570 and VDR rs731236 between type 2 DM patients and controls [49], no contribution of VDR rs1544410 to type $1 \mathrm{DM}$ susceptibility [50], and no association of $V D R$ rs1544410 with chronic kidney disease susceptibility [51].

In this study we were not able to show significant differences in the frequency distribution of tested polymorphic variants of T-cell-related cytokine genes or vitamin D pathway genes between HD patients with ESRD due to type 2 DM nephropathy and controls as well as HD patients with other causes of ESRD analyzed together. This lack of association was present although the examined type $2 \mathrm{DM}$ nephropathy patients showed clinical complications more frequently than HD patients with other renal diseases: higher dialysis related mortality rate [3], higher prevalence of CAD including myocardial infarction [4], lower serum PTH, and lower frequency of parathyroidectomy and treatment with cinacalcet, all of them predictive for higher tendency to adynamic bone disease [7]. Type 2 DM nephropathy patients with or without diagnosis of CAD also did not differ in tested genotype distributions.

Development of ESRD substantially ameliorates interpatient clinical variability related to underlying renal impairment and exposes uremia-related signs and symptoms. Comparisons of type $2 \mathrm{DM}$ nephropathy patients in respect of tested genotype frequencies with subjects showing other common causes of ESRD revealed that the former group has a higher IL18 rs360719 minor allele frequency than chronic infective tubulointerstitial nephritic group. In this case, lower IL18 rs360719 minor allele frequency in tubulointerstitial nephritic patients was observed also when their results were compared to those of healthy subjects. Sánchez et al. [52] have found a significant increase in the relative expression of IL18 mRNA in individuals carrying the rs360719 minor allele. IL-18 is IFN- $\gamma$ inducing factor. Infective tubulointerstitial nephritic patients are known to have diminished ability of blood leukocytes to produce IFN- $\gamma$ [53]. Our study indicates that this may be related to lower frequency of IL18 rs360719 minor allele in this group compared to controls and type 2 DM nephropathy patients. In type 2 DM patients with overt nephropathy, positive correlations between plasma IFN- $\gamma$, proteinuria, and eGFR were found [54].

Due to limited financial support, we did not perform any functional studies regarding T-cell-related interleukin and vitamin $\mathrm{D}$ pathway genes, especially that multiple influences independent or dependent on genetic profile need to be taken into account in such studies conducted in the uremic milieu. Although the examined patients showing ESRD due to type 2 DM nephropathy were well-defined group, they obviously were not consistent in HLA DRB1 alleles. The latter could be important in modulating susceptibility to advanced type 2 DM nephropathy and related complications, like it was shown for type $1 \mathrm{DM}$ [55] or type $2 \mathrm{DM}$ [41], regardless of their complications.

\section{Summary}

Distributions of tested T-cell cytokine gene polymorphisms or vitamin D pathway gene polymorphisms are not significantly different among patients with ESRD due to type 2 DM nephropathy and healthy individuals. Subjects with ESRD due to type $2 \mathrm{DM}$ nephropathy differ in clinical manifestation from patients with other nephropathies leading to dialysis dependency, but differences in tested genotype distributions were found only in IL18 rs360719 compared with chronic tubulointerstitial nephritic patients. This difference probably arose from the fact that pathology of chronic infective tubulointerstitial nephritis might have been associated with this specific polymorphism.

\section{Conclusions}

In Polish HD patients, T-cell cytokine gene polymorphisms and vitamin D pathway gene polymorphisms are not associated with ESRD due to type 2 DM nephropathy. IL18 polymorphism is worthy to be further investigated in chronic infective tubulointerstitial nephritic patients as being possibly associated with this disease.

\section{Conflict of Interests}

The authors declare that there is no conflict of interests regarding the publication of this paper.

\section{References}

[1] E. Villar, H. C. Sean, and S. P. McDonald, "Incidences, treatments, outcomes, and sex effect on survival in patients with end-stage renal disease by diabetes status in Australia and New Zealand (1991-2005)," Diabetes Care, vol. 30, no. 12, pp. 30703076, 2007.

[2] A. Sattar, C. Argyropoulos, L. Weissfeld et al., "All-cause and cause-specific mortality associated with diabetes in prevalent hemodialysis patients," BMC Nephrology, vol. 13, article 130, 2012.

[3] F. Chantrel, I. Enache, M. Bouiller et al., "Abysmal prognosis of patients with type 2 diabetes entering dialysis," Nephrology Dialysis Transplantation, vol. 14, no. 1, pp. 129-136, 1999.

[4] H. Al-Thani, A. Shabana, A. Hussein et al., "Cardiovascular complications in diabetic patients undergoing regular hemodialysis: a 5-year observational study," Angiology, 2014.

[5] M. J. Sarnak and B. L. Jaber, "Mortality caused by sepsis in patients with end-stage renal disease compared with the general population," Kidney International, vol. 58, no. 4, pp. 1758-1764, 2000.

[6] S.-M. Alavian and S. V. Tabatabaei, "The effect of diabetes mellitus on immunological response to hepatitis $B$ virus vaccine in individuals with chronic kidney disease: a meta-analysis of current literature," Vaccine, vol. 28, no. 22, pp. 3773-3777, 2010. 
[7] D. Zayour, M. Daouk, W. Medawar, M. Salamoun, and G. ElHajj Fuleihan, "Predictors of bone mineral density in patients on hemodialysis," Transplantation Proceedings, vol. 36, no. 5, pp. 1297-1301, 2004.

[8] D. M. Li, Y. Zhang, B. Ding et al., "The association between vitamin $\mathrm{D}$ deficiency and diabetic nephropathy in type 2 diabetic patients," Zhonghua Nei Ke Za Zhi, vol. 52, no. 11, pp. 970-974, 2013 (Chinese).

[9] C.-C. Wu, H.-K. Sytwu, K.-C. Lu, and Y.-F. Lin, "Role of T cells in type 2 diabetic nephropathy," Experimental Diabetes Research, vol. 2011, Article ID 514738, 9 pages, 2011.

[10] Y. Tian, C. Wang, Z. Ye, X. Xiao, A. Kijlstra, and P. Yang, "Effect of 1,25-Dihydroxyvitamin D3 on Th17 and Th1 response in patients with Behçet's disease," Investigative Ophthalmology and Visual Science, vol. 53, no. 10, pp. 6434-6441, 2012.

[11] J. Stachowski, "Hepatitis C virus infection in renal diseases: state of knowledge, therapeutic problems and perspectives," Polski Merkuriusz Lekarski, vol. 8, no. 46, pp. 303-306, 2000 (Polish).

[12] B. D. Livingston, J. Alexander, C. Crimi et al., "Altered helper T lymphocyte function associated with chronic hepatitis $\mathrm{B}$ virus infection and its role in response to therapeutic vaccination in humans," Journal of Immunology, vol. 162, no. 5, pp. 3088-3095, 1999.

[13] J. Zhang, G. Hua, X. Zhang, R. Tong, X. Du, and Z. Li, "Regulatory $\mathrm{T}$ cells/T-helper cell 17 functional imbalance in uraemic patients on maintenance haemodialysis: a pivotal link between microinflammation and adverse cardiovascular events," Nephrology, vol. 15, no. 1, pp. 33-41, 2010.

[14] E. Borella, G. Nesher, E. Israeli, and Y. Shoenfeld, "Vitamin D: a new anti-infective agent?" Annals of the New York Academy of Sciences, vol. 1317, no. 1, pp. 76-83, 2014.

[15] E. Zitt, H. Sprenger-Mähr, F. Knoll, U. Neyer, and K. Lhotta, "Vitamin D deficiency is associated with poor response to active hepatitis B immunisation in patients with chronic kidney disease," Vaccine, vol. 30, no. 5, pp. 931-935, 2012.

[16] T. Shoji and Y. Nishizawa, "Vitamin D and survival of hemodialysis patients," Clinical calcium, vol. 14, no. 9, pp. 64-68, 2004 (Japanese).

[17] L. Steingrimsdottir, O. Gunnarsson, O. S. Indridason, L. Franzson, and G. Sigurdsson, "Relationship between serum parathyroid hormone levels, vitamin D sufficiency, and calcium intake," The Journal of the American Medical Association, vol. 294, no. 18, pp. 2336-2341, 2005.

[18] R. Pacifici, "Role of T cells in the modulation of PTH action: physiological and clinical significance," Endocrine, vol. 44, no. 3, pp. 576-582, 2012.

[19] H. Zhang, J. Wang, B. Yi et al., "BsmI polymorphisms in vitamin $\mathrm{D}$ receptor gene are associated with diabetic nephropathy in type 2 diabetes in the Han Chinese population," Gene, vol. 495, no. 2, pp. 183-188, 2012.

[20] N. Mtiraoui, I. Ezzidi, M. Kacem et al., "Predictive value of interleukin-10 promoter genotypes and haplotypes in determining the susceptibility to nephropathy in type 2 diabetes patients," Diabetes/Metabolism Research and Reviews, vol. 25, no. 1, pp. 5763, 2009.

[21] I. Ezzidi, N. Mtiraoui, M. Kacem et al., "Interleukin-10-592C/A, $-819 \mathrm{C} / \mathrm{T}$ and $-1082 \mathrm{~A} / \mathrm{G}$ promoter variants affect the susceptibility to nephropathy in Tunisian type 2 diabetes (T2DM) patients," Clinical Endocrinology, vol. 70, no. 3, pp. 401-407, 2009.

[22] M. Karadeniz, M. Erdogan, A. Berdeli, and C. Yilmaz, "Association of interleukin-6 -174 G>C promoter polymorphism with increased risk of type 2 diabetes mellitus patients with diabetic nephropathy in Turkey," Genetic Testing and Molecular Biomarkers, vol. 18, no. 1, pp. 62-65, 2014.

[23] M. Murea, T. C. Register, J. Divers et al., "Relationships between serum MCP-1 and subclinical kidney disease: African American-Diabetes Heart Study," BMC Nephrology, vol. 13, no. 1, article 148, 2012.

[24] A. E. Grzegorzewska, D. Pajzderski, A. Sowińska, and P. P. Jagodziński, "Polymporphism of monocyte chemoattractant protein 1 (MCP1-2518 A/G) and responsiveness to hepatitis B vaccination in hemodialysis patients," Polskie Archiwum Medycyny Wewnetrznej, vol. 124, no. 1-2, pp. 10-18, 2014.

[25] A. E. Grzegorzewska, D. Pajzderski, A. Sowińska, and P. P. Jagodziński, "Monocyte chemoattractant protein-1 gene (MCP1-2518 A/G) polymorphism and serological markers of hepatitis B virus infection in hemodialysis patients," Medical Science Monitor, vol. 20, pp. 1101-1116, 2014.

[26] Group KDIGOKCW, "KDIGO 2012 clinical practice guideline for the evaluation and management of chronic kidney disease," Kidney International Supplements, vol. 3, pp. 1-150, 2013.

[27] A. E. Grzegorzewska, P. Wobszal, and P. P. Jagodziński, "Interleukin-18 promoter polymorphism and development of antibodies to surface antigen of hepatitis B virus in hemodialysis patients," Kidney and Blood Pressure Research, vol. 35, no. 1, pp. 1-8, 2012.

[28] A. E. Grzegorzewska, P. M. Wobszal, A. Sowińska, A. Mostowska, and P. P. Jagodziński, "Association of the interleukin-12 polymorphic variants with the development of antibodies to surface antigen of hepatitis B virus in hemodialysis patients in response to vaccination or infection," Molecular Biology Reports, vol. 40, no. 12, pp. 6899-6911, 2013.

[29] A. E. Grzegorzewska, P. M. Wobszal, A. Mostowska, and P. P. Jagodziński, "Antibodies to hepatitis B virus surface antigen and interleukin 12 and interleukin 18 gene polymorphisms in hemodialysis patients," BMC Nephrology, vol. 13, no. 1, article 75, 2012.

[30] A. E. Grzegorzewska, D. Pajzderski, A. Sowińska, A. Mostowska, and P. P. Jagodziński, "IL4R and IL13 polymorphic variants and development of antibodies to surface antigen of hepatitis $\mathrm{B}$ virus in hemodialysis patients in response to $\mathrm{HBV}$ vaccination or infection," Vaccine, vol. 31, no. 14, pp. 1766-1770, 2013.

[31] S. S. Rich, "Genetics of diabetes and its complications," Journal of the American Society of Nephrology, vol. 17, no. 2, pp. 353-360, 2006.

[32] N. D. Palmer and B. I. Freedman, "Insights into the genetic architecture of diabetic nephropathy," Current Diabetes Reports, vol. 12, no. 4, pp. 423-431, 2012.

[33] N. Franceschini, N. M. Shara, H. Wang et al., "The association of genetic variants of type 2 diabetes with kidney function," Kidney International, vol. 82, no. 2, pp. 220-225, 2012.

[34] N. D. Palmer, C. W. McDonough, P. J. Hicks et al., "A genomewide association search for type 2 diabetes genes in african americans," PLoS ONE, vol. 7, no. 1, Article ID e29202, 2012.

[35] A. Davoodi-Semiromi, J. J. Yang, and J.-X. She, "IL-12p40 is associated with type 1 diabetes in Caucasian-American families," Diabetes, vol. 51, no. 7, pp. 2334-2336, 2002.

[36] D. B. Mirel, A. M. Valdes, L. C. Lazzeroni, R. L. Reynolds, H. A. Erlich, and J. A. Noble, "Association of IL4R haplotypes with type 1 diabetes," Diabetes, vol. 51, no. 11, pp. 3336-3341, 2002.

[37] T. L. Bugawan, D. B. Mirel, A. M. Valdes, A. Panelo, P. Pozzilli, and H. A. Erlich, "Association and interaction of the IL4R, 
IL4, and IL13 loci with type 1 diabetes among filipinos," The American Journal of Human Genetics, vol. 72, no. 6, pp. 15051514, 2003.

[38] B. Frederiksen, E. Liu, J. Romanos et al., "Investigation of the vitamin D receptor gene (VDR) and its interaction with protein tyrosine phosphatase, non-receptor type 2 gene (PTPN2) on risk of islet autoimmunity and type 1 diabetes: the Diabetes Autoimmunity Study in the Young (DAISY)," The Journal of Steroid Biochemistry and Molecular Biology, vol. 133, no. 1, pp. 51-57, 2013.

[39] C. Panierakis, G. Goulielmos, D. Mamoulakis, E. Petraki, E. Papavasiliou, and E. Galanakis, "Vitamin D receptor gene polymorphisms and susceptibility to type 1 diabetes in Crete, Greece," Clinical Immunology, vol. 133, no. 2, pp. 276-281, 2009.

[40] Q. Wang, B. Xi, K. H. Reilly, M. Liu, and M. Fu, "Quantitative assessment of the associations between four polymorphisms (FokI, ApaI, BsmI, TaqI) of vitamin D receptor gene and risk of diabetes mellitus," Molecular Biology Reports, vol. 39, no. 10, pp. 9405-9414, 2012.

[41] N. M. Al-Daghri, O. Al-Attas, M. S. Alokail et al., "Vitamin D receptor gene polymorphisms and HLA DRB $* 04$ cosegregation in Saudi type 2 diabetes patients," Journal of Immunology, vol. 188, no. 3, pp. 1325-1332, 2012.

[42] F.-L. Vélayoudom-Céphise, L. Larifla, J.-P. Donnet et al., "Vitamin D deficiency, vitamin D receptor gene polymorphisms and cardiovascular risk factors in Caribbean patients with type 2 diabetes," Diabetes \& Metabolism, vol. 37, no. 6, pp. 540-545, 2011.

[43] D. A. F. Ferrarezi, N. Bellili-Muñoz, D. Dubois-Laforgue et al., "Allelic variations of the vitamin D receptor (VDR) gene are associated with increased risk of coronary artery disease in type 2 diabetics: the DIABHYCAR prospective study," Diabetes and Metabolism, vol. 39, no. 3, pp. 263-270, 2013.

[44] T. B. Zhou, Z. P. Jiang, M. F. Huang, and N. Su, "Association of vitamin D receptor Fok1 (rs2228570), TaqI (rs731236) and ApaI (rs7975232) gene polymorphism with the risk of chronic kidney disease," Journal of Receptors and Signal Transduction Research, vol. 5, pp. 1-5, 2014.

[45] R. D. Mittal and P. K. Manchanda, "Association of interleukin (IL)-4 intron-3 and IL-6 -174 G/C gene polymorphism with susceptibility to end-stage renal disease," Immunogenetics, vol. 59, no. 2, pp. 159-165, 2007.

[46] G. Morahan, E. McKinnon, J. Berry et al., "Evaluation of IL12B as a candidate type I diabetes susceptibility gene using data from the Type I Diabetes Genetics Consortium," Genes and Immunity, vol. 10, supplement 1, pp. S64-S68, 2009.

[47] H. A. Erlich, K. Lohman, S. J. MacK et al., "Association analysis of SNPs in the IL4R locus with type i diabetes," Genes and Immunity, vol. 10, supplement 1, pp. S33-S41, 2009.

[48] R. Jorde, H. Schirmer, T. Wilsgaard et al., "Polymorphisms related to the serum 25-Hydroxyvitamin D level and risk of Myocardial infarction, diabetes, cancer and mortality. The Tromsø study," PLoS ONE, vol. 7, no. 5, Article ID e37295, 2012.

[49] H. Bid, R. Konwar, C. Aggarwal et al., "Vitamin D receptor (FokI, BsmI and TaqI) gene polymorphisms and type 2 diabetes mellitus: a North Indian study," Indian Journal of Medical Sciences, vol. 63, no. 5, pp. 187-194, 2009.

[50] M. C. Lemos, A. Fagulha, E. Coutinho et al., "Lack of association of vitamin D receptor gene polymorphisms with susceptibility to type 1 diabetes mellitus in the Portuguese population," Human Immunology, vol. 69, no. 2, pp. 134-138, 2008.
[51] T. B. Zhou, Z. P. Jiang, and M. F. Huang, "Association of vitamin D receptor BsmI (rs1544410) gene polymorphism with the chronic kidney disease susceptibility," Journal of Receptors and Signal Transduction Research, 2014.

[52] E. Sánchez, R. J. Palomino-Morales, N. Ortego-Centeno et al., "Identification of a new putative functional IL18 gene variant through an association study in systemic lupus erythematosus," Human Molecular Genetics, vol. 18, no. 19, pp. 3739-3748, 2009.

[53] I. P. Kudriashova, T. P. Ospel'nikova, and F. I. Ershov, "Cycloferon administration in chronic pyelonephritis: changes in interferon status," Terapevticheskil Arkhiv, vol. 83, no. 6, pp. 33-35, 2011 (Russian).

[54] C.-C. Wu, J.-S. Chen, K.-C. Lu et al., "Aberrant cytokines/chemokines production correlate with proteinuria in patients with overt diabetic nephropathy," Clinica Chimica Acta, vol. 411, no. 9-10, pp. 700-704, 2010.

[55] N. Israni, R. Goswami, A. Kumar, and R. Rani, "Interaction of Vitamin D receptor with HLA DRB $* 0301$ in Type 1 diabetes patients from North India," PLoS ONE, vol. 4, no. 12, Article ID e8023, 2009. 


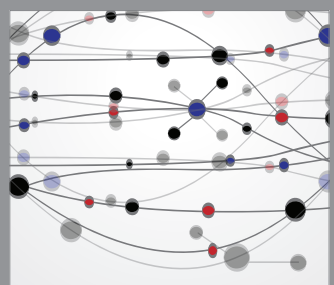

The Scientific World Journal
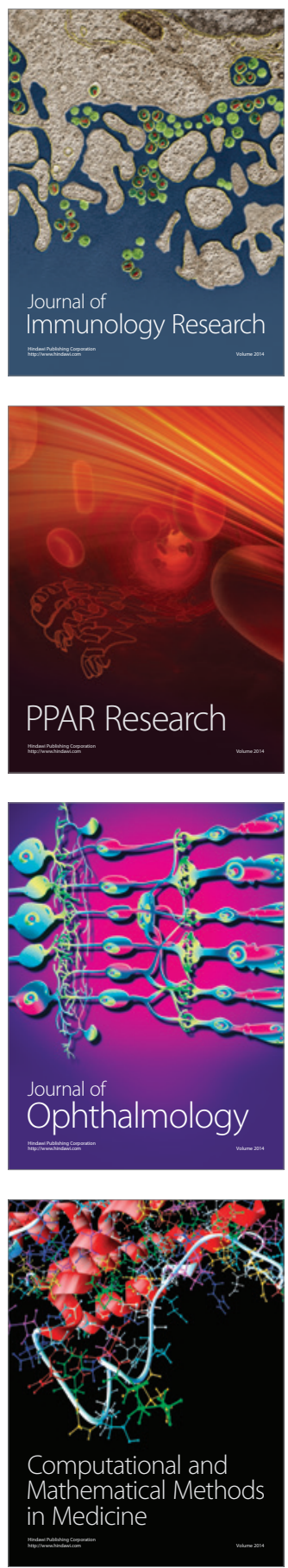

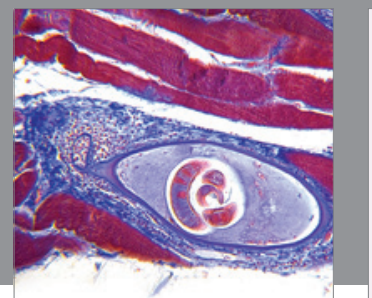

Gastroenterology

Research and Practice
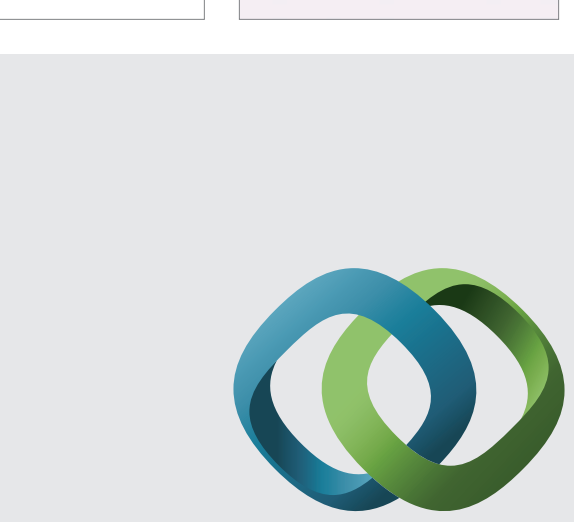

\section{Hindawi}

Submit your manuscripts at

http://www.hindawi.com
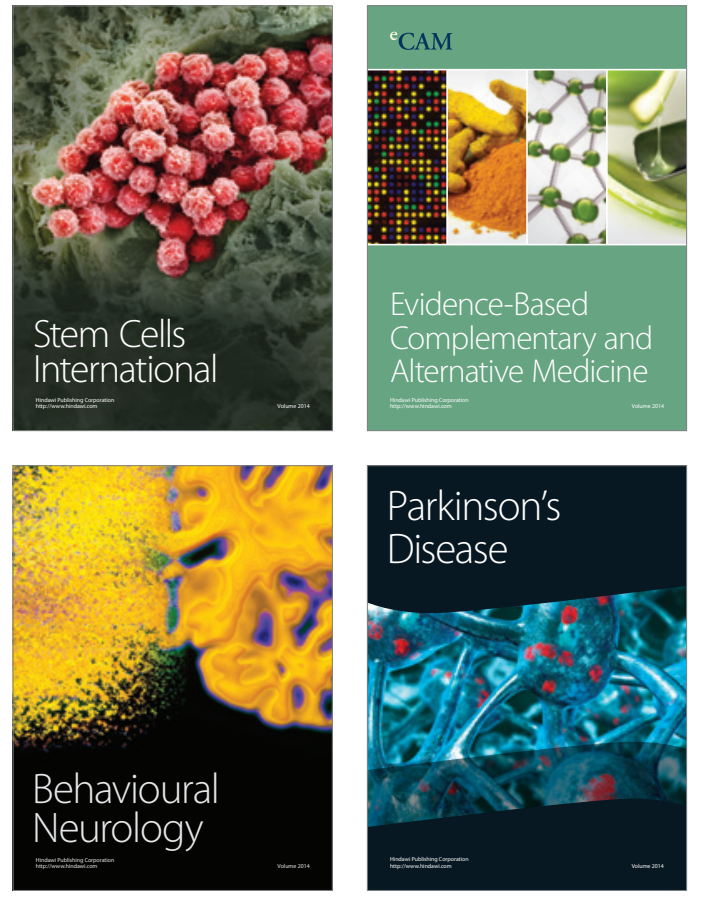
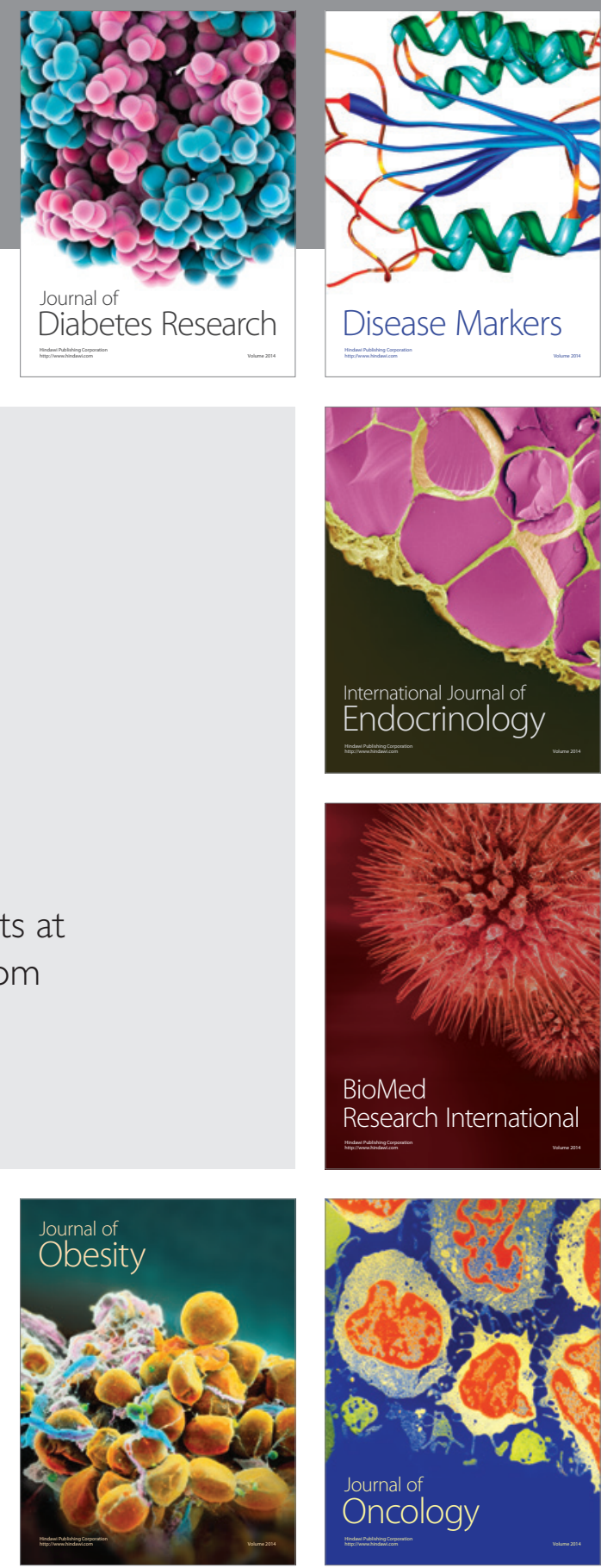

Disease Markers
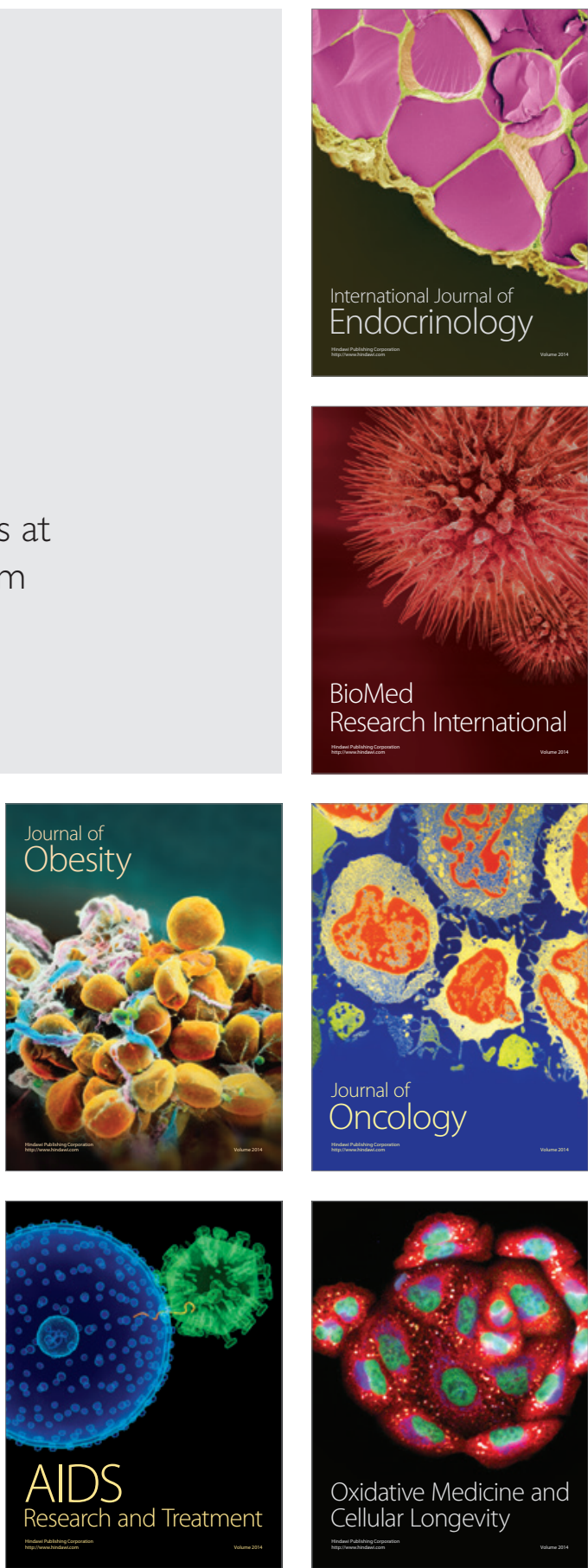